\title{
Develop Techniques for Ion Implantation of PLZT for Adaptive Optics
}
C. R. Batishko
K. A. Stahl
J. L. Brimhall
L. H. Toburen
W. T. Pawlewicz

September 1987

Prepared for

the U.S. Department of the Air Force

Air Force Weapons Laboratory

under a Related Services Agreement

with the U.S. Department of Energy

under Contract DE-AC06-76RLO 1830

Pacific Northwest Laboratory

Operated for the U.S. Department of Energy

by Battelle Memorial Institute 


\title{
DISCLAIMER
}

This report was prepared as an account of work sponsored by an agency of the United States Government. Neither the United States Government nor any agency thereof, nor Battelle Memorial Institute, nor any of their employees, makes any warranty, expressed or implied, or assumes any legal liability or responsibility for the accuracy, completeness, or usefulness of any information, apparatus, product, or process disclosed, or represents that its use would not infringe privately owned rights. Reference herein to any specific commercial product, process, or service by trade name, trademark, manufacturer, or otherwise, does not necessarily constitute or imply its endorsement, recommendation, or favoring by the United States Government of any agency thereof, or Battelle Memorial Institute. The views and opinions of authors expressed herein do not necessarly state or reflect those of the United States Government or any agency thereof, or Battelle Memorial Institute.

\author{
PACIFIC NORTHWEST LABORATORY \\ operated by \\ BATTELLE MEMORIAL INSTITUTE \\ for the \\ UNITED STATES DEPARTMENT OF ENERGY \\ under Contract DE-AC06-76RLO 1830
}


DEVELOP TECHNIQUES FOR

ION IMPLANTATION OF PLZT

FOR ADAPTIVE OPTICS
C. R. Batishko
J. L. Brimhall
W. T. Pawlewicz
K. A. Stahl
L. H. Toburen

July 1987

Prepared for

the Department of the Air Force

Air Force Weapons Laboratory under a Related Services Agreement with the U. S. Department of Energy

Contract DE-AC06-76RLO 1830

Pacific Northwest Laboratory

Richland, Washington 99352 



\section{PREFACE}

This project was initiated in the second quarter of FY85 with a primary objective of providing the sponsor with implanted PLZT samples to use in his experimental adaptive optics work. FY85 was characterized by a learning process during which the hardware was adapted and characterization capabilities developed. Samples implanted were intended to demonstrate capability by duplicating work done previously at Sandia by Peercy and Land. The fiscal year closed with the implantation capability having been successfulty demonstrated, but with few samples delivered.

The FY86 objectives were considerably more ambitious, and characterized by a reed to deliver usable samples. Coatings task leader W. T. Pawlewicz was added to the project team and charged with developing an optimum ITO coating, investigating stress cracking hypothesized as being due to the ITO coating mechanical properties mismatch, and attempting to fabricate thin film PLZT.

Characterization task objectives emphasized duplicating the characterization capabilities at Sandia so that comparisons could be made, but also defined some areas in which additional characterization would be attempted.

This report is broken into sections written by task leaders Batishko, Brimhal1, Pawlewicz, and Toburen, and is intended to provide an overview of the year's work, with selected data illustrating the most significant of the work. Tables of samples produced and delivered are included. 



\section{SUMMARY}

In fiscal years 1985 and 1986, the Pacific Northwest Laboratory (PNL) conducted research into the preparation and characterization of ion implanted adaptive optic elements based on lead-lanthanum-zirconate-titanate (PLZT) technology. The objectives for FY 1985 were to acquire and/or adapt the necessary hardware and acquire the experience needed to implant PLZT following procedures provided by the sponsor and Dr. Cecil Land of Sandia National Laboratory.

The objectives for FY86 were to continue to develop the capability to ion implant and coat PLZT, initiate implantations which would enhance the photosensitivity and extend it to longer wavelengths, investigate stress behavior which leads to sample cracking, prepare samples for delivery to the sponsor for use in his research, and characterize the wafers for application as adaptive optical elements.

At the end of FY86, accomplishments reflecting the status of the project include: 1) a total of 25 samples have been prepared and delivered to the sponsor for his research, 2) a total of nine different species or combinations of species have been implanted, 3) stress cracking has been investigated and found to not be due to the ITO electrode coating as originally hypothesized, 4) a means of deducing implantation layer refractive index, thickness, and density from transmission measurements was developed, and 5) a preliminary experiment was conducted to deposit thin film PLZT. Detailed results and data on the implantation process, characterization of the implanted and electroded samples, and on the various stress experiments is included in the report.

This report concludes with a brief summary of proposed FY86 tasks. 



\section{ABSTRACT}

Research was conducted at Pacific Northwest Laboratory to develop high photosensitivity adaptive optical elements utilizing ion implanted lanthanumdoped lead-zirconate-titanate (PLZT). One centimeter square samples were prepared by implanting ferroelectric and anti-ferroelectric PLZT with a variety of species or combinations of species. These included $\mathrm{Ne}, \mathrm{O}, \mathrm{Ni}, \mathrm{Ne} / \mathrm{Cr}, \mathrm{Ne} / \mathrm{AT}$, $\mathrm{Ne} / \mathrm{Ni}, \mathrm{Ne} / \mathrm{O}$, and $\mathrm{Ni} / \mathrm{O}$, at a variety of energies and fluences.

An indium-tin oxide (ITO) electrode coating was designed to give a balance of high conductivity and optical transmission at near UV to near IR wavelengths.

Samples were characterized for photosensitivity; implanted layer thickness, index of refraction, and density; electrode (ITO) conductivity; and in some cases, residual stress curvature.

Thin film anti-ferroelectric PLZT was deposited in a preliminary experiment. The structure was amorphous with $x$-ray diffraction showing the beginnings of a structure at substrate temperatures of approximately $550^{\circ} \mathrm{C}$.

This report summarizes the research and provides a sampling of the data taken during the report period. 


\section{CONTENTS}

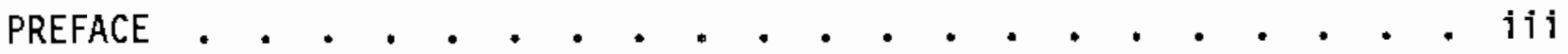

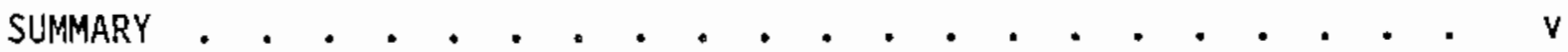

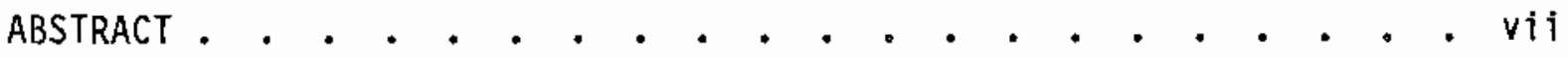

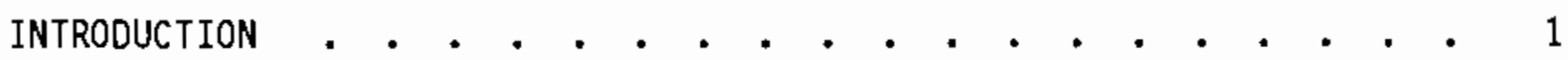

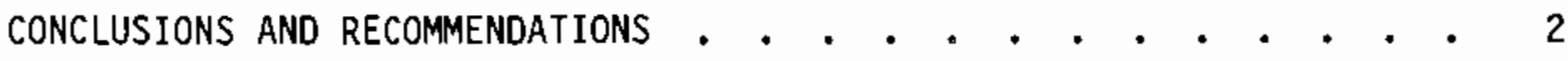

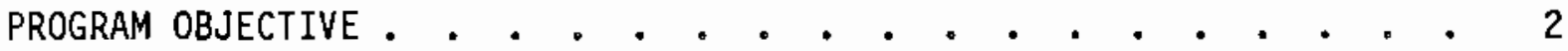

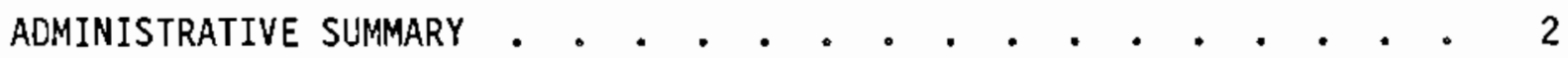

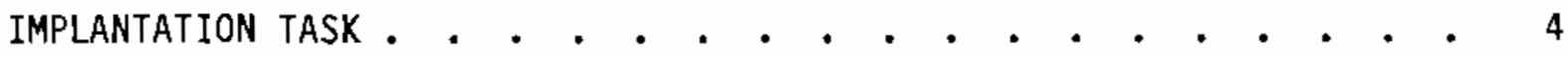

SUMMARY • • • • • • . . . . . . . . . • . . . . 4

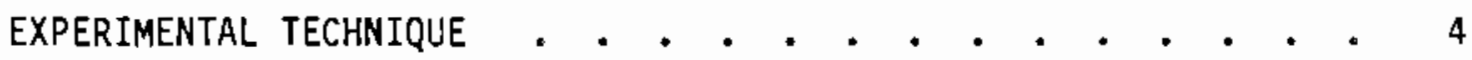

CALCULATION OF IMPLANTATION DAMAGE $\quad \cdot \quad \cdot \quad \cdot \quad \cdot \quad \cdot \quad \cdot \quad \cdot \quad \cdot \quad 7$

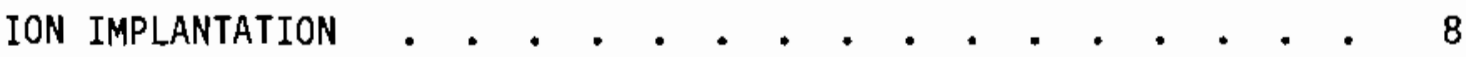

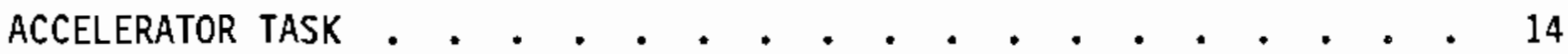

COATINGS TASK • . . . . . . . . . . . . . . . . . . 15

ITO COATINGS: PREPARATION AND CHARACTERIZATION $\quad$ • • • • 15

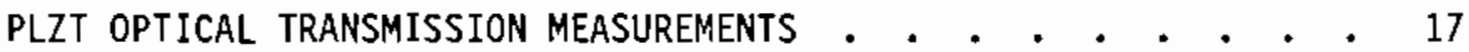

IMPLANTED PLZT REFRACTIVE INDEX, DENSITY AND THICKNESS • • • 17

PLZT CURVATURE AND MECHANICAL STRESS . . . • . . . • . * . 21

PLZT CURVATURE VERSUS APPLIED VOLTAGE - • • • • • • • • • 26

PRELIMINARY THIN-FILM PLZT FABRICATION EXPERIMENTS . • • • • 30

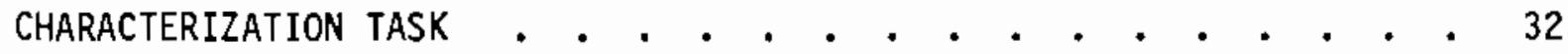

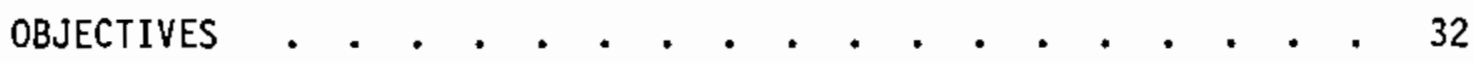

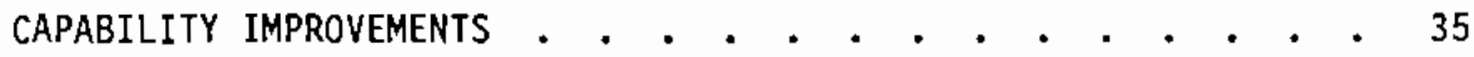

TRANSMISSION MEASUREMENTS . . . . . . . • . . . . . . 37

PHOTOSENSITIVITY MEASUREMENTS • • • • • • • • • • • • • • 44 
CONTENTS (continued)

PROPOSED FY87 TASKS . . . . . . . . . . . . . . . . 55

REFERENCES . . . . . . . . . . . . . . . . . 64 


\section{FIGURES}

1 Time-Phased Spending Plan . . . . . . . . . . . . . 3

2 Project Milestone Chart . . . . . . . . . . . . . . 5

3 Sample Holder Configuration . . . . . . . . . . . 6

4 Damage Curves for Implantation of $\mathrm{Ne}^{+}$and $\mathrm{Ni}^{+}$Ions for Sample 9/13 A-C . . . . . . . . . . . . . . . . . . 11

5 Damage Curves for Implantation of $\mathrm{Ne}^{+}$and $\mathrm{O}^{+}$Ions for Sample

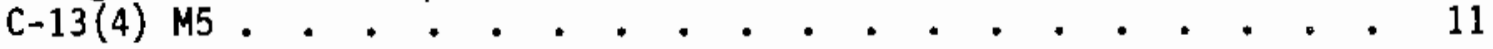

6 Damage Curves for Implantation of $\mathrm{Ne}^{+}$and $\mathrm{Al}^{+}$Ions for Samples $7 / 25$ A-D, $7 / 24$ A,B . . . . . . . . . . . . . . . 12

7 Damage Curves for Implantation of $\mathrm{Ne}^{+}$and $\mathrm{Cr}^{+}$Ions for Samples $7 / 24$ C, D, 7/23 A-D . . . . . . . . . . . . . . . 12

8 Damage Curves for Variable Energy Implant of $\mathrm{Ni}^{+}$Ions for Samples 9/20 A,B,D . . . . . . . . . . . . . . . . . 13

9 IR Transmission . . . . . . . . . . . . . . . 18

10 PLZT Wafer After Implant . . . . . . . . . . . . 24

11 Implanted Sample Curvature Hysteresis . . . . . . . . . . 27

12 ITO Stress $\left(10^{8}\right.$ dynes $\left./ \mathrm{cm}^{2}\right)$. . . . . . . . . . . . . . 28

13 Thin Film PLZT Transmission . . . . . . . . . . . . . 31

14 Thin Film PLZT X-Ray Diffraction . . . . . . . . . . . 33

15a Sample Photographed in Transmission . . . . . . . . . 36

15b Interferogram . . . . . . . . . . . . . . 36

Diffraction of $3 \mathrm{~mW}$ HeNe Beam by 300 Line/Inch Ronchi Ruling
Printed on Sample $9 / 18 \mathrm{~B}$

17 Diffraction of $25 \mathrm{~mW}$ HeNe Beam by 300 Line/Inch Ronchi Ruling Printed on Sample 9/19B . . . . . . . . . . . . 38

18 Transmission, As-Received Wafer . . . . . . . . . . 39

19 Transmission, Arnealed Wafer . . . . . . . . . . . . 40

20 Transmission, Cut and Annealed Wafer . . . . . . . . 41 


\section{FIGURES (continued)}

21 Transmission, Implanted, ITO Coated Sample . . . . . . . . 42

22 Transmission, As-Received 7/70/30 Wafer . . . . . . . . 43

23 Hysteresis Curve, Oxygen Implanted Sample . . . . . . . . 45

24a 50 Line/Inch Ronchi Ruling Printed on Sample B-8 . . . . . . 46

24b Interferogram of 24a . . . . . . . . . . . . . . . 46

25 Hysteresis Curves, Oxygen/Nickel, $476.2 \mathrm{~nm}$. . . . . . . . 47

26 Hysteresis Curves, Oxygen/Nickel, $476.2 \mathrm{~nm}$, Expanded . . . . . 48

27 Hysteresis Curves, Neon/Nickel, $476.2 \mathrm{~nm}$. . . . . . . . . 49

28 Hysteresis Curves, Neon/Nickel, $482.5 \mathrm{~nm}$. . . . . . . . . 50

29 Hysteresis Curves, Neon/Nickel, 520.B nm . . . . . . . . 51

30 Sensitivity Curves, Neon/Nickel and Diffused Aluminum/Neon . . . 52

31 Sensitivity Curves, Noen/Nickel and Land's Sample . . . . . . 53

32 Hysteresis Curves, Aluminum/Nickel, $476.2 \mathrm{~nm}$. . . . . . . 54

33 Spectral Sensitivity, Neon/Aluminum in 7/70 PLZT . . . . . . 56

34 Hysteresis Curves, Neon/Aluminum in 7/70 PLZT, After Re-Anneal • 57

35 Spectral Sensitivity, Neon/Aluminum in 7/70 PLZT, After Re-Anneal 58

36 Hysteresis Curves, Chromium/Neon in 7/70 PLZT . . . . . . . 59

37 Spectral Sensitivity, Chromium/Neon in 7/70 PLZT . . . . . . 60

38 Hysteresis Curves, Multiple Nitrogen, $476.2 \mathrm{~nm}$. . . . . . . 61

39 Hysteresis Curves, Multiple Nitrogen, $676.4 \mathrm{~nm}$. . . . . . . 62 


\section{$\underline{\text { TABLES }}$}

1 Ion Implantations in FY 1986 . . . . . . . . . . . . . 9

2 Sample Irradiations . . . . . . . . . . . . . . 16

3 PLZT Implanted Layer Characterization . . . . . . . . 20

4 PLZT Curvature (Mechanical Stress) versus Processing Step . . . 23

5 Reduction of Implant-Induced Wafer Curvature by $200^{\circ} \mathrm{C} / 10$ Hour/ Air Anneal . • . • • • • . . • . • • • 25

6 XES Compositional Analysis of 9065 PLZT Target and F1lms . . . 34

7 FY87 Proposed Tasks . . . . . . . . . . . . . . . 63 


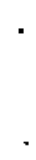




\section{DEVELOP TECHNIQUES FOR ION IMPLANTATION}

OF PLZT FOR ADAPTIVE OPTICS

\section{INTRODUCTION}

optical beams propagating through an inhomogeneous medium are aberrated by point-to-point phase differences across the wavefront, resulting in decreased efficiency in transmitting a focused beam through the medium. By introducing equal and opposite phase differences into the transmitted beam before propagation, medium-induced phase differences will exactiy cancel those introduced at transmission resulting in formation of a diffraction limited focal spot. Near real-time measurement of the medium-induced phase differences and subsequent correction may be accomplished with optical phase conjugation utilizing adaptive optical components fabricated from ion implanted PLZT. A sampling beam passing through the medium interferes with a reference beam at the PLZT wafer to form a hologram which stores medium-induced phase shifts. The beam to be transmitted is used to reconstruct the hologram, introducing the compensating phase shifts, and resulting in a diffraction limited focal spot at the sample beam position.

The United States Air Force Weapons Laboratory at Kirtland Air Force Base is funding this research at the Pacific Northwest Laboratory (PNL) in support of the development of the PLZT adaptive optical components needed for phase correction.

This research has focused on the ion implantation of PLZT to enhance the photosensitivity at wavelengths of interest. In the process, related areas of implantation-induced stress cracking, optically transparent electrode design, and optical characterization of resulting samples have been addressed.

This report is an annual progress report which summarizes the research during fiscal year 1986. It describes the processing of PLZT samples for a range of implantation species and parameters and presents representative data characterizing those samples. Finally, it presents conclusions reached by analyzing and interpreting the data. In recommending the continuation of the project, a set of objectives for fiscal year 1987 are presented. 


\section{CONCLUSIONS AND RECOMMENDATIONS}

Through the course of the year, a number of conclusions were reached on various aspects of the program. These are as follows:

- oxygen implants seem to show enhanced and flattened spectral photosensitivities.

- the stress cracking earlier hypothesized to be due to a mismatch between ITO and PLZT mechanical properties appears to be due entirely to implantation-induced damage.

- producing thin film PLZT of the correct crystalline structure will requjre elevated substrate temperatures and carefully controlled target composition.

- We recommend that funds be authorized and the project be continued through FY87. We recommend that the implantation species and process parameters continue to be investigated, and that an additional task be added to the scope, to develop a model in support of the continued research. In addition, we recommend increased emphasis on development of the thin film PLZT deposition process, and subsequent implantation.

\section{PROGRAM OBJECTIVE}

The objective of this program is to develop techniques for the fabrication of adaptive optical components wth high photosensitivity using ion implantation of Lanthanum-doped Lead Zirconate Titanate (PLZT) and sputtered transparent electrodes, and to evaluate the samples produced for spectral photosensitivity, contrast, and resolving power.

\section{ADMINISTRATIVE SUMMARY}

The funding level for this project under Amendment 1 to the original proposal was $\$ 175,000$. Figure 1 shows a time phased spending plan with both estimated spending profile and actual spending shown. At the end of FY85, approximately $\$ 5,300$ remained unspent. It was anticipated that this amount 
FIGURE 1

\section{AFWL Adaptive Optics Project}

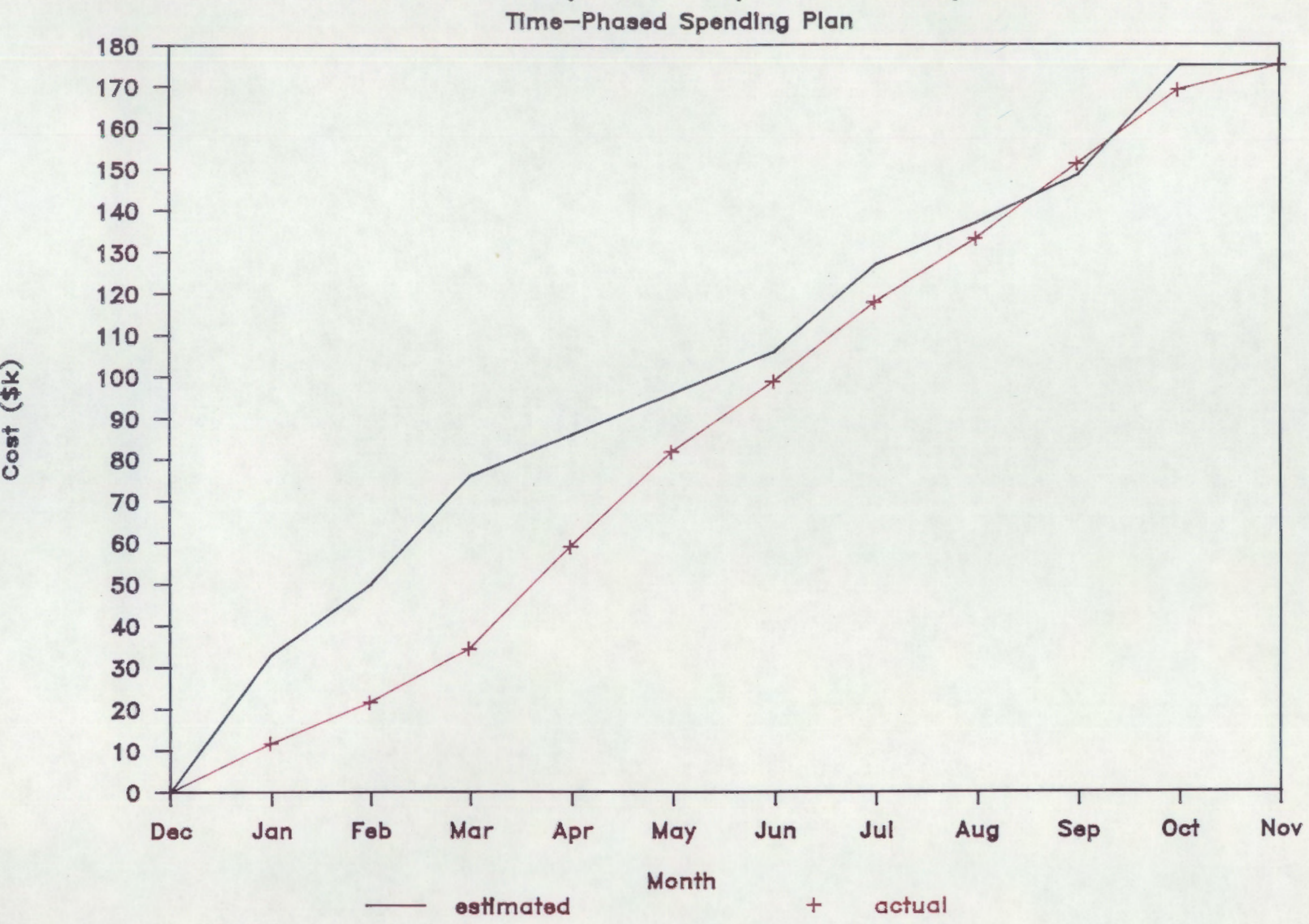


would be adequate to complete work in progress and prepare the annual progress report.

Figure 2 shows a milestone chart for FY86. At the conclusion of FY86, all milestones have been met with the exception of those postponed to allow higher priority activities, installation of the bipolar power supply delayed by procurement delays and changing priorities, and the delivery of this report.

Late in the fiscal year, Dr. Richard A. Craig was invited to join the team contingent on definition of goals and funding for FY87. Dr. Craig will lead a theory/modeling task to assist in more efficiently selecting implant species for enhanced photosensitivity. As an introduction to the project, Dr. Craig participated in the year-end review at PNL.

\section{IMPLANTATION TASK}

\section{SUMMARY}

Approximately 50 specimens of PLZT have been ion implanted during FY86. Ions of neon, aluminum, chromium, nickel, nitrogen, and oxygen in various combinations have been used. Both the type of ion and ion energy have been varied in the multiple ion implants. In this report, the technique used in the implantations is described, the expected damage distribution from the implantations is calculated, and a listing of all implants and the parameters is presented.

\section{EXPERIMENTAL TECHNIQUE}

Prior to implantation, the $1^{\prime \prime}$ square sheet of PLZT is cut into four smaller sheets $0.5^{\prime \prime}$ on a side. One side is coated with a thin ( $\left.10 \mathrm{~nm}\right)$ layer of ITO to dissipate charge build-up during implantation. Six specimens are mounted on a copper block as shown in Figure 3 and held in place by clamping at opposite corners. The copper block is mounted on a rotatable shaft with vertical and horizontal motion as well.

During implantation, the beam is aligned through the hole in the copper block; this hole is exactly centered on the 1" $\times 1$ " face of the copper block. Once the maximum current is aligned through the hole, the holder is rotated $45^{\circ}$ 


\section{FIGURE 2}

- us air force Wezoons lab PLZT ADAPTIVE OPTICS PROJECT

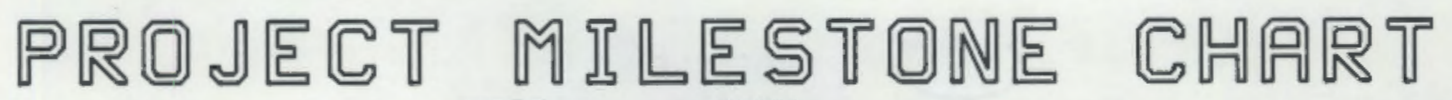

\begin{tabular}{|c|c|c|c|c|c|c|c|c|c|c|c|c|c|}
\hline manth & $\begin{array}{l}1985 \\
\text { OCT }\end{array}$ & Nov & DEC & $\begin{array}{c}1986 \\
\text { JPAN }\end{array}$ & FEB & MRR & |APR & MAY & JUN & JuL & RUG & SEP & LCT \\
\hline AUTHORIZATION & & $\nabla$ & & & & & & & & & & & \\
\hline & & & & & & & & & & & & & \\
\hline \multicolumn{14}{|l|}{ IMPLANTATION } \\
\hline $1 \mathrm{MeV} \mathrm{O+}$ & $\bar{\nabla}$ & & & & & & & & & & & & \\
\hline Install Ne beam line & & & 8 & & & & & & & & & & \\
\hline $500 \mathrm{KeV} \mathrm{Net}$ & & & $\nabla$ & & & & & & & & & & \\
\hline $\mathrm{Cr}+\mathrm{Ne}$, diffused $\mathrm{Al}+\mathrm{Ne}$ & & & & $=$ & & $\nabla \ldots$ & $\bar{\ldots}$ & $\ldots$. & 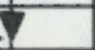 & & & & \\
\hline FFE $\mathrm{Cr}+\mathrm{Ne} \& \mathrm{Al}+\mathrm{Ne}$ & & & & & & & & & & & 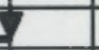 & & \\
\hline multiple energy $\mathrm{N} 1$ & & & & & & & & & & & & & 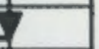 \\
\hline $1.2 \mathrm{MeV} \mathrm{N}+$ & & & & & & & & & & $\bar{\nabla}$. & & Y. & . \\
\hline $\mathrm{Ta}$ and/or $\mathrm{Au}+\mathrm{Ne}$ & & & & & & & & & $\nabla$. & $\ldots y$ & $7 \ldots$ & $\ldots \ldots$ & $\ldots$ \\
\hline $9 / 65 / 35$ AFE & & & & & & ... & ...... & .... & $\ldots$ & $\ldots$. & $\ldots$ & $\ldots \ldots$ & $\ldots \ldots$ \\
\hline & & & & & & & & & & & & & \\
\hline COATINGS & & & & & & & & & & & & & \\
\hline select coating design specs. & & $=$ & 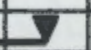 & & & & & & & & & & \\
\hline optimize coating process & & 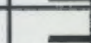 & & 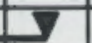 & & & & & & & & & \\
\hline measure mechanical stress & & & & & & & & & & & & & \\
\hline stress-optimized coating & & & & & & & & & 1 & & & & \\
\hline thin film PLZT fab attempt & & & & & & & & & & & & 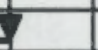 & \\
\hline coat samples as needed & & - & & & & & & & & & & & $\frac{1}{1}$ \\
\hline characterize $\% T$ and resistivity & & - & & & & & & & & & & ta & 4 \\
\hline & & & & & & & & & & & & & \\
\hline CHARACTERIZATION & & & & & & & & & & & & & \\
\hline install/test fon laser & & $\bar{\nabla}$ & & & & & & & & & & & \\
\hline assamble interferometer & & & & $\nabla$ & & & & & & & & & \\
\hline procure substrates & & - & & $\mathbf{7}$ & & & & & & & & & \\
\hline Install bipolar power supply & & & & & & & $\frac{1}{7} \ldots$ & & & & $\frac{1}{7} \ldots$ & & \\
\hline modify software & & & & & & & 5 & & & & & & \\
\hline contrast measurement & & & & & & & & & 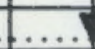 & $\frac{1}{7}$ & & & \\
\hline sensitivity me asurement & & & & & & & & 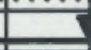 & & & & & \\
\hline deliver implanted samples & & & & & $\nabla$ & & & $\overline{7}$ & & $\frac{t}{t}$ & $\nabla$ & & $\frac{1}{y}$ \\
\hline & & & & & & & & & & & & & \\
\hline MFNAGEMENT & & & & & & & & & & & & & \\
\hline progress reports & & & & & & & & & & 7 & & 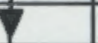 & \\
\hline review meeting & & & & & $\nabla$. & .7 & & & $\bar{\nabla}$ & & & $\bar{\nabla}$ & \\
\hline tech. info, exchange $\mathrm{mtg}$. & & & & & & $\nabla$. & .7 & & & & $\nabla$ & & \\
\hline . annual report & & & & & & & & & & & $D$ & F. & 支. \\
\hline
\end{tabular}




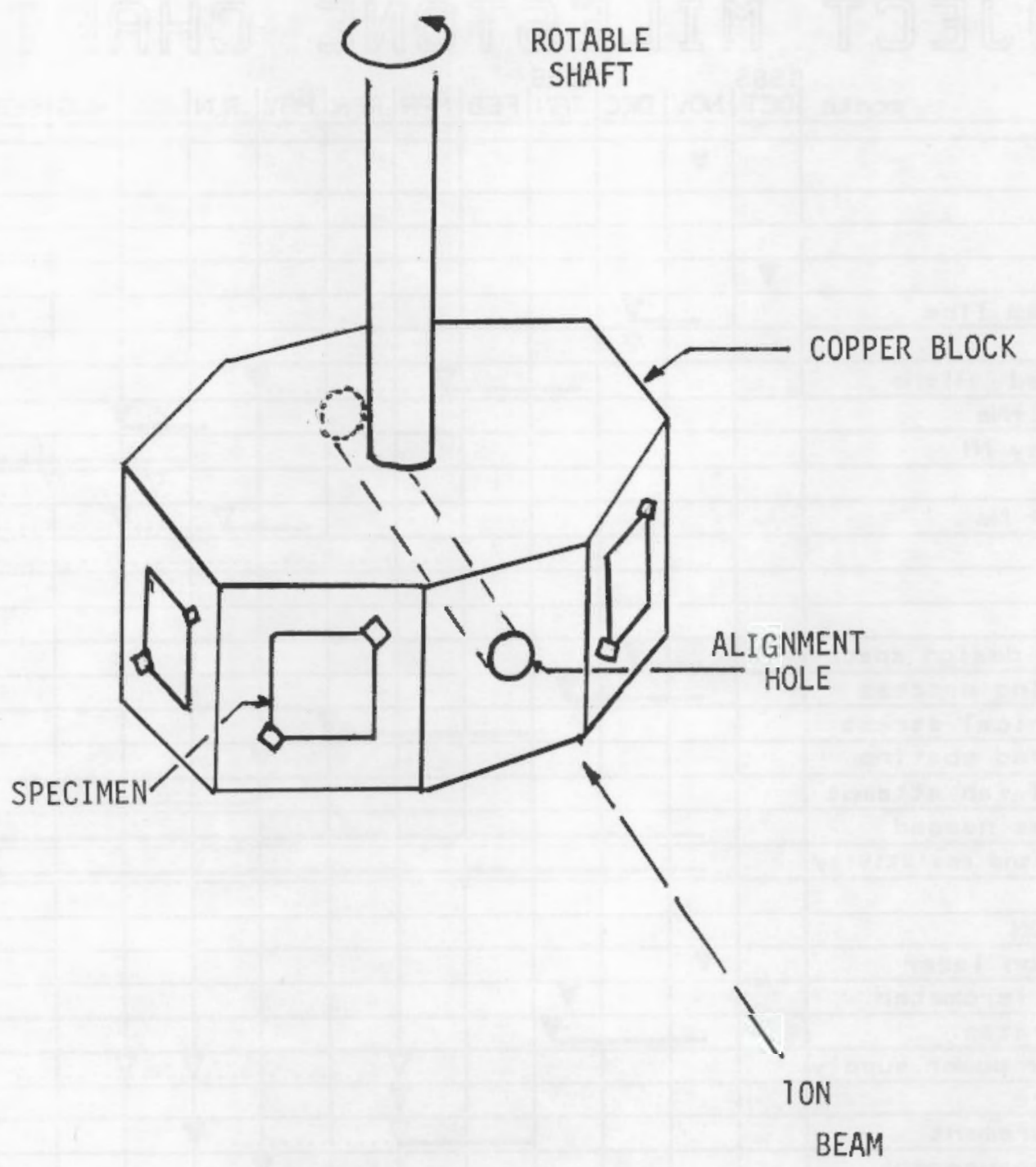

FIGURE 3. Sample Holder Configuration 
or multiple thereof to bring the specimen into the beam. An estimate of beam size is made by comparing the current through the 0.25 " diameter hole to the current in a $0.5^{\prime \prime}$ diameter faraday cap.

Beam lines from two separate accelerators are brought into the specimen chamber so a variety of implants can be done without moving the specimen from the vacuum chamber. Inert gas ions are accelerated up to $\sim 1.8 \mathrm{n} \mathrm{MeV} \mathrm{with} \mathrm{a} \mathrm{van}$ der Graaff accelerator. A $2 \mathrm{MeV}$ tandem accelerator is used for all other ions. Depending on the charge state of the ions, energies up to $8 \mathrm{MeV}$ can be obtained on the tandem. The vacuum in the specimen chamber is typically $\left\langle 1 \times 10^{-7}\right.$ torr when using the tandem, but only in the $10^{-6}$ torr range when using the van der Graaff.

During implantation, the beam is rastered in a serpentine fashion across the surface to provide uniformity of implantation. The beam current is also monitored on the specimen during irradiation. The sample holder is not suppressed for electron scatter, so the current will read higher than actual beam current. The beam current is checked periodically with the faraday cup located behind the holder and this is used to get the integrated dose on the specimen. The beam can also be observed visually in the darkened chamber due to fluorescence from the specimen. Beam current densities ranged from $0.02 \mu \mathrm{amp} / \mathrm{cm}^{2}$ to $0.5 \mu \mathrm{amp} / \mathrm{cm}^{2}$. The surface temperature was monitored periodically by an infrared pyrometer when using high beam currents. The temperature was always less than $160^{\circ} \mathrm{C}$, the minimal temperature measured by the pyrometer.

\section{CALCULATION OF IMPLANTATION DAMAGE}

The damage distribution produced by the ions is calculated using the EDEP program. This program determines the energy deposited into the material during the ion-atom interaction. Both electronic and nuclear energy losses are calculated. It is only the nuclear energy loss that produces displaced atoms. The number of displaced atoms (dpa) can be computed from the following expression: 


$$
d p a=\frac{\beta S D(x)(\phi t)}{T_{D} N_{D}}
$$

$S D(x)=$ nuclear energy loss in $\mathrm{MeV} / \mu \mathrm{m}$

$\mathrm{T}_{D} \quad=$ energy required to permanently displace atom from its site $\sim 40 \mathrm{e.v}$.

$\beta \quad=$ numerical factor $\sim 2$

$\Phi \mathrm{t} \quad=$ ion fluence in ions $/ \mathrm{cm}^{2}$

$\mathrm{N}_{\mathrm{D}} \quad=$ atomic density in atoms $/ \mathrm{cm}^{3}$

The dpa value gives a measure of the magnitude of the damage produced in the lattice in terms of atom defects. The range and range straggling of the ions are also computed in the E-DEP program.

The concentration of deposited ions can be calculated from the expression:

$$
\begin{aligned}
& c_{i}=\frac{(\phi t)}{2 \pi \sigma} \exp \frac{(x-\bar{x})^{2}}{2 \sigma^{2}} \\
& c_{i}= \text { atom concentration of deposited ions at point } x \\
& \Phi t=\text { ion fluence } \\
& \sigma=\text { standard deviation of the distribution of deposited ion } \\
& \bar{x}=\text { projected range of ions }
\end{aligned}
$$

This assumes a Gaussian distribution with a maximum at the projected range. For ion fluences of $1 \times 10^{15}$ and energies near $1 \mathrm{MeV}$, the maximum concentrations are $\langle 0.1 \%$. The standard deviation $\sigma$ is a direct function of the ion penetration so high energy ions will have a large $\sigma$ and hence a broader distribution.

\section{ION IMPLANTATIONS}

A list of the implantations accomplished during FY86 is shown in Table 1. Representative damage curves calculated from the EDEP program using actual implantation conditions are shown in Figures 4 through 8 . All the curves show a maximum near the end of the damage region. The range of the ions $\bar{x}$ is always 
Table I - Ion Implantations in FY-1986

No.

Specimen

Implant

Dose

of Specimens

(1)

$1.4 \mathrm{MeV} 0^{+}$

$3 \times 10^{15}$

1

\#4 $A_{1} B$

$500 \mathrm{KeV} \mathrm{Ne}^{+}$

$1 \times 10^{15}$

2

(impure beam)

\#5 (A-D)

Diffused A1 (vac)

$500 \mathrm{KeV} \mathrm{Ne}^{+}$

$1 \times 10^{15}$

4

$C-13(1,2,3)$

$500 \mathrm{KeV} \mathrm{Ne}{ }^{+}$

$1 \times 10^{15}$

3

$1.4 \mathrm{MeV} \mathrm{Ni}^{+}$

$1 \times 10^{15}$

C-13(4), M5)

$500 \mathrm{KeV} \mathrm{Ne}^{+}$

$1.4 \mathrm{MeV} \mathrm{O}^{+}$

$1 \times 10^{15}$

2

$2 \times 10^{15}$

$B 7(1,2,3)$

$1.4 \mathrm{MeV} \mathrm{O}^{+}$

$2 \times 10^{15}$

$1.4 \mathrm{MeV} \mathrm{Ni}^{+}$

$1 \times 10^{15}$

$9 / 22(A-D)$

Diffused $A 1$ in Air

$+500 \mathrm{KeV} \mathrm{Ne}{ }^{+}$

$1 \times 10^{15}$

4

$9 / 13(A-C)$

$500 \mathrm{KeV} \mathrm{Ne}^{+}$

$1 \times 10^{15}$

$900 \mathrm{KeV} \mathrm{Ne}^{+}$

$1.5 \times 10^{15}$

3

$1.4 \mathrm{MeV} \mathrm{Ni}^{+}$

$5 \times 10^{14}$

9-13D

$500 \mathrm{KeV} \mathrm{Ne}{ }^{+}$

$1 \times 10^{15}$

9-14A, B

$900 \mathrm{KeV} \mathrm{Ne}^{+}$

$1.4 \mathrm{MeV} \mathrm{Ni}^{+}$

$1.5 \times 10^{15}$

$7 \times 10^{14}$

3

$7 / 25(A-D)$

$1.3 \mathrm{MeV} \mathrm{Ne}^{+}$

$2.5 \times 10^{15}$

6

$7 / 24(A, B)$

$1.3 \mathrm{MeV} \mathrm{Al}^{+}$

$1 \times 10^{15}$ 
Table 1 - Ion Implantations in FY-1986 (Continued)

$\begin{array}{lllc}\text { Specimen } & \text { Implant } & \text { Dose } & \begin{array}{c}\text { No. } \\ \text { of Specimens }\end{array} \\ 7 / 24 \mathrm{C}, \mathrm{D} & 1.3 \mathrm{MeV} \mathrm{Ne}^{+} & 2 \times 10^{15} & 5 \\ 7 / 23, \mathrm{~A}-\mathrm{D} & 1.4 \mathrm{MeV} \mathrm{Cr} & 8 \times 10^{14} & \\ & & & \\ 9 / 20 \mathrm{~A}, \mathrm{~B}, \mathrm{D} & 5 \mathrm{MeV} \mathrm{Ni}^{++} & 1 \times 10^{15} & 3 \\ & 2.4 \mathrm{MeV} \mathrm{Ni}^{+} & 4 \times 10^{14} & 3 \\ & 1.3 \mathrm{MeV} \mathrm{Ni}^{+} & 2.5 \times 10^{14} & \\ & & & \\ 9 / 21 \mathrm{~A}, \mathrm{~B}, \mathrm{C} & 4.5 \mathrm{MeV} \mathrm{Ni}^{++} & 5 \times 10^{14} & \\ & 2 \mathrm{MeV} \mathrm{Ni}^{+} & 2 \times 10^{14} & 3 \\ & 1.3 \mathrm{MeV} \mathrm{Ni}^{+} & 1.25 \times 10^{14} & \end{array}$




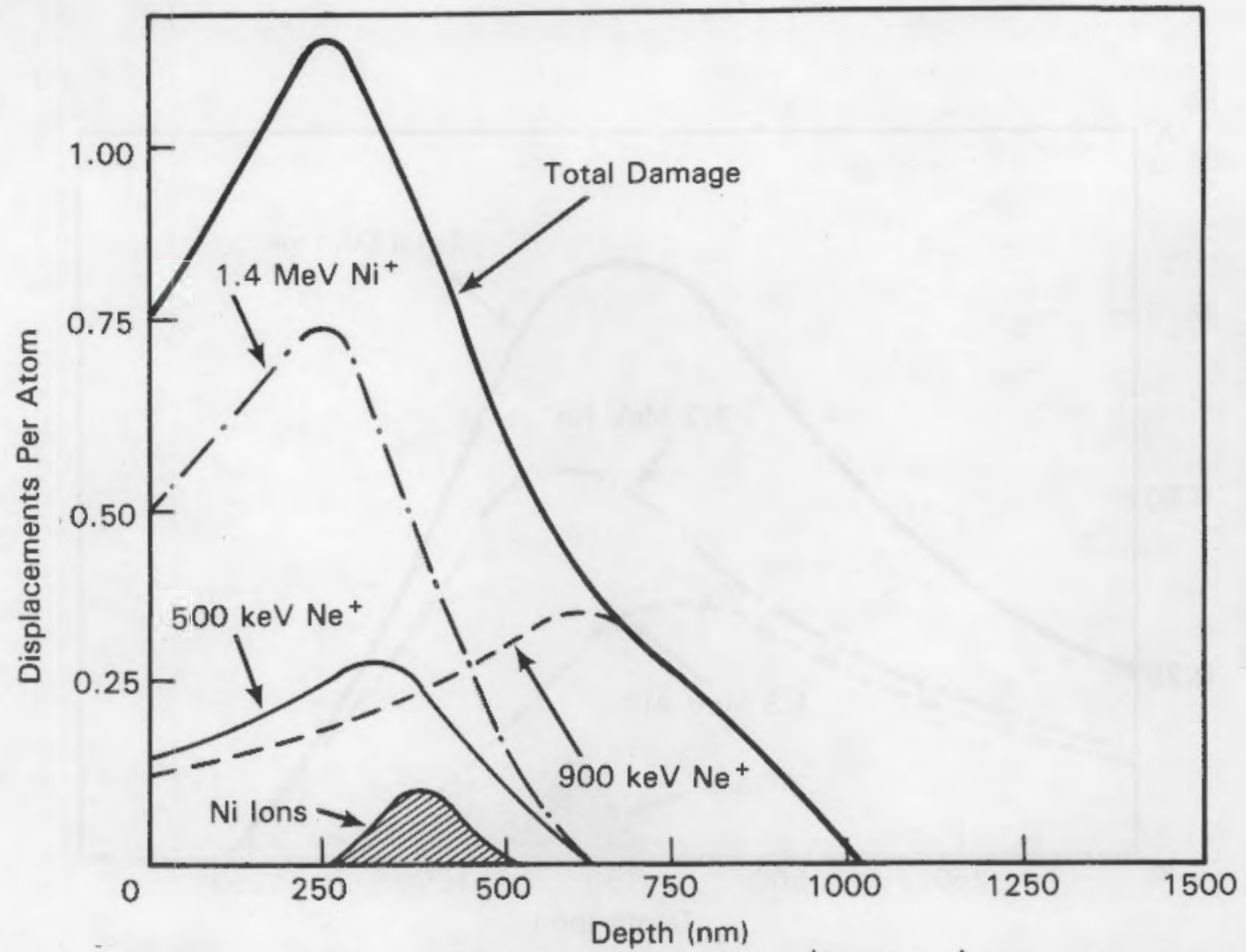

Figure 4. Damage Curves for Implantation of $\mathrm{Ne}^{+}$and $\mathrm{Ni}^{+}$ions for Sample 9/13 A-C.

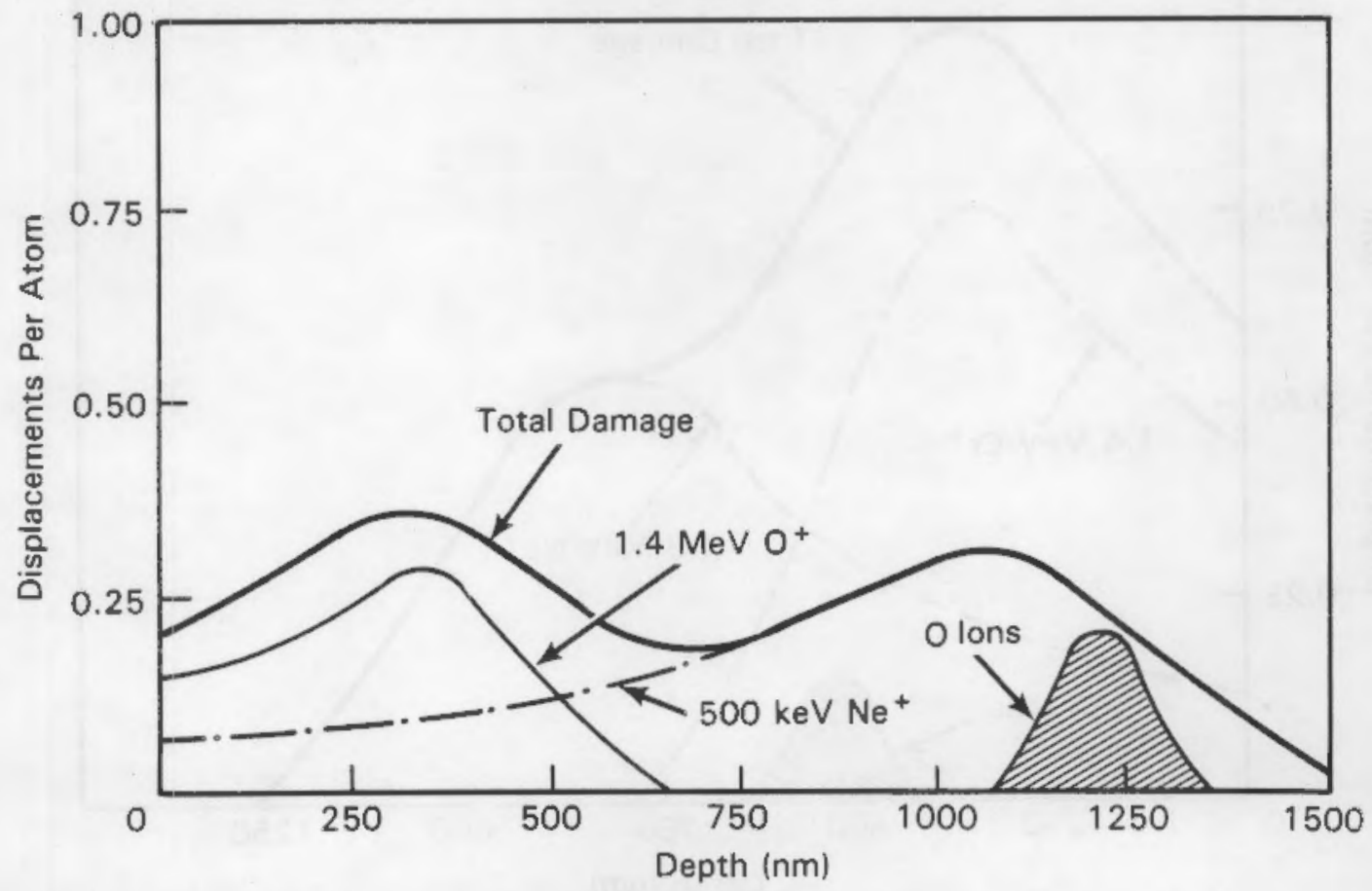

Figure 5. Damage Curves for Implantation of $\mathrm{Ne}^{+}$and $\mathrm{O}^{+}$ions for Samples C-13(4) M5. 


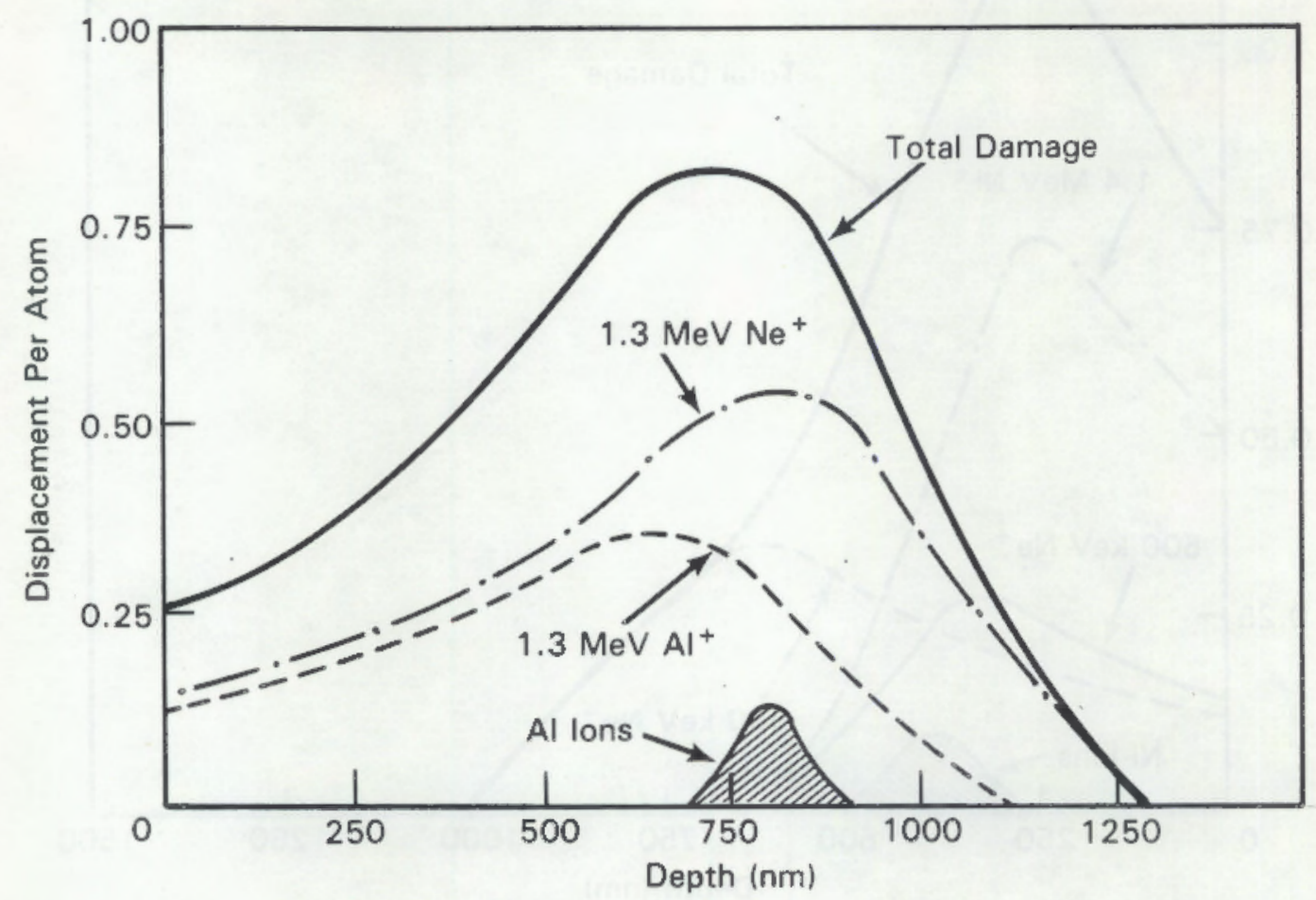

Figure 6. Damage Curves for Implantation of $\mathrm{Ne}^{+}$and $\mathrm{Al}^{+}$ions for Samples $7 / 25$ A-D, 7/24 A,B.

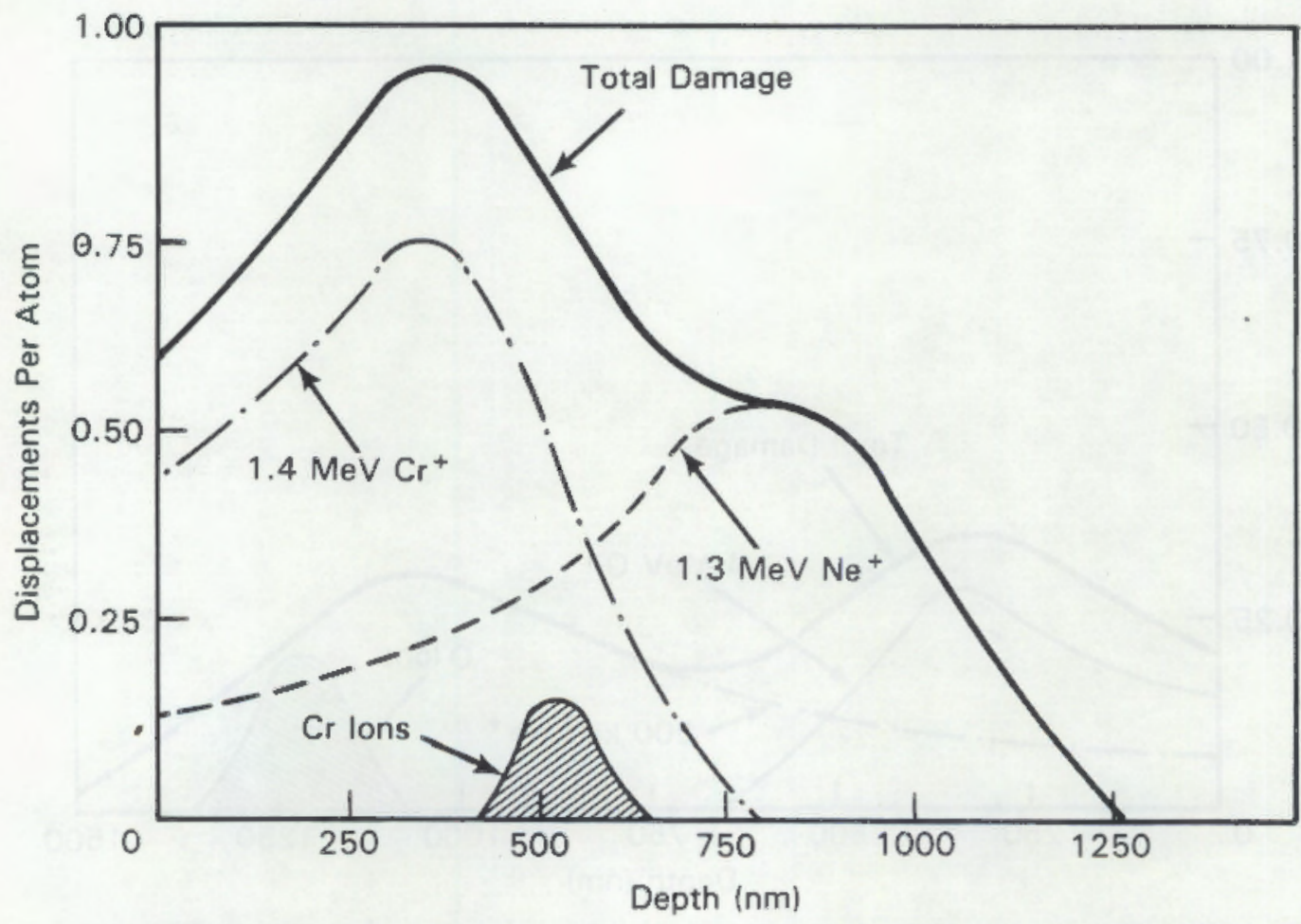

Figure 7. Damage Curves for Implantation of $\mathrm{Ne}^{+}$and $\mathrm{Cr}^{+}$ions for Samples $7 / 24$ C,D, $7 / 23$ A-D. 


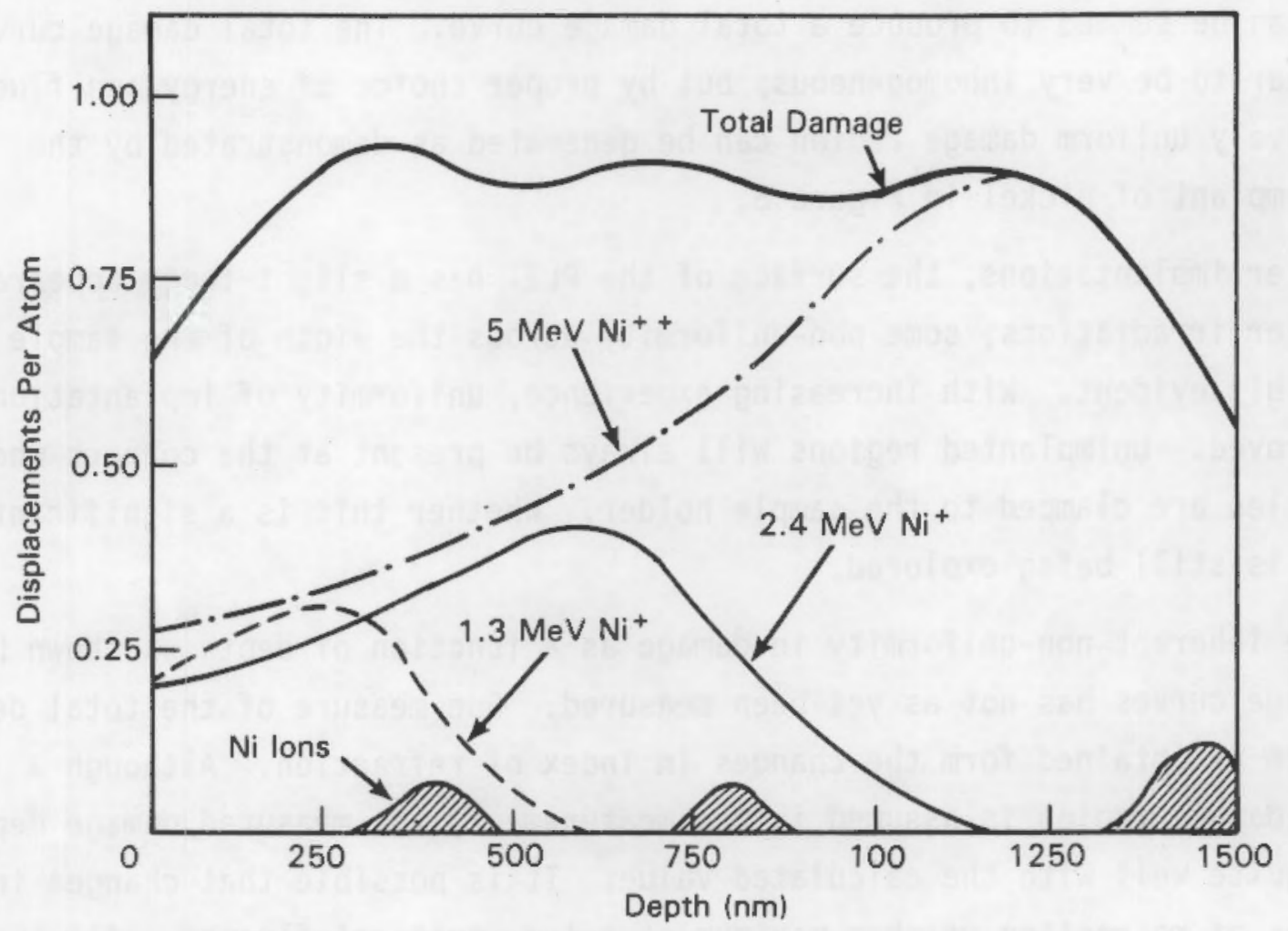

Figure 8. Damage Curves for Variable Energy Implant of $\mathrm{Ni}^{+}$ions for Samples 9/20 A, B, D. 
just beyond the maximum in the damage curve. As shown by the curves, lighter ions will give a greater depth of damage but less damage per unit fluence than heavier ions for comparable ion energies. For multiple implants, the individual curves can be summed to produce a total damage curve. The total damage curves can appear to be very inhomogeneous; but by proper choice of energy and fluence, a relatively uniform damage region can be generated as demonstrated by the triple implant of nickel in Figure 8.

After implantations, the surface of the PLZT has a slight foggy appearance. In earlier irradiations, some non-uniformity across the width of the sample was visibly evident. With increasing experience, uniformity of implantation has improved. Unimplanted regions will always be present at the corners where the samples are clamped to the sample holder. Whether this is a significant problem is still being explored.

The inherent non-uniformity in damage as a function of depth as shown by the damage curves has not as yet been measured. One measure of the total depth of damage is obtained form the changes in index of refraction. Although a uniform damage region is assumed in the measurement, the measured damage depth agrees quite well with the calculated value. It is possible that changes in the index of refraction reach a maximum at a low, critical fluence. All the doses used so far in the experiments may be greater than this critical fluence, so that variations in damage distribution are not detected.

\section{ACCELERATOR TASK}

The accelerator task is separate from the implantation task, primarily for organizational convenience and accounting purposes. The work within the task is in most cases inseparable from the implantation task. One of the areas which can be identified primarily with this task was the preparation, installation, and testing of a velocity selector for use in separating ion species when one of the beams appeared to be overly contaminated. Installation was done early in the year, but the contamination was related to the bottle of gas in use, and was resolved by installing a new bottle. The device was not tested or used until late in the year when the nitrogen implant appeared to 
require better separation of species in the beam. At that time, it was still not certain that the selector would or could resolve the problem.

The other part of this task which is clearly separable is the operation of the accelerators in support of implantation. Table 2 contains a list of the irradiations performed this fiscal year in support of the program. of particular interest is the relative times for various species. The amount of implantation time required per unit area of a sample depends on the beam current which can be generated for that species. Of those species implanted this year, aluminum gave the lowest beam current and thus required the longest beam times.

\section{COATINGS TASK}

\section{ITO COATINGS: PREPARATION AND CHARACTERIZATION}

Indium Tin Oxide (ITO) coatings were applied by dc magnetron reactive sputtering techniques (Pawlewicz 1986) in a 28-inch box chamber outfitted with a planetary substrate holder. An air-cast metal target (20 weight $\%$ tin) was sputtered in an $\mathrm{Ar} / \mathrm{O}_{2}$ atmosphere. The optically monitored transmission loss with increasing thickness was used to control stoichiometry so that sheet resistance was held at the minimum value. The PLZT wafer temperature was about $40^{\circ} \mathrm{C}$ during coating application.

ITO coating thickness was always $160 \mathrm{~nm}$ (three quarter waves at the 425 $\mathrm{nm}$ monitoring wavelength). Sheet resistance measured by the 4-point van der Pauw method (van der Pauw 1958) averaged 85 ohms/square and was always within the range 60 to 130 ohms/square. Coating both sides, on the average, maintained $91 \%$ of the implanted PLZT transmission at $425 \mathrm{~nm}$ and a higher percentage (greater than $100 \%$ in some cases) at longer wavelengths.

Some PLZT wafers received a thin ITO coating before implantation to dissipate static charge. These coatings were about $20 \mathrm{~nm}$ thick with sheet resistances of about 1000 ohms/square. The thin coatings were optically monitored for a transmission drop from 92 to $80 \%$ at $425 \mathrm{~nm}$ on fused silica wetness substrates. This thickness just exceeded that required for electrical continuity. 
TABLE 2. Sample Irradiations

\begin{tabular}{|c|c|c|c|}
\hline DATE & BEAM/ENERGY & ACCELERATOR & IIME \\
\hline 25-NOV-1985 & 1.4 MeV 0xygen +1 & Tandem & $3 \mathrm{Hrs}$. \\
\hline 3-JAN-1986 & 1.0 MeV Neon & VDG & $1 \mathrm{Hr}$. \\
\hline 7-JAN-1986 & $.5 \mathrm{MeV}$ Neon & VDG & 2 Hrs. \\
\hline 23-JAN-1986 & $.5 \mathrm{MeV}$ Neon & VDG & 4 Hrs. \\
\hline 10-FEB-1986 & $.5 \mathrm{MeV}$ Neon & VDG & 4 Hrs. \\
\hline 13-FEB-1986 & 1.4 MeV Nickel +1 & Tandem & $8 \mathrm{Hrs}$. \\
\hline 25-FEB-1986 & 1.4 MeV Oxygen +1 & Tandem & $7 \mathrm{Hrs}$. \\
\hline 28-FEB-1986 & 1.4 MeV Nickel +1 & Tandem & $6 \mathrm{Hrs}$. \\
\hline 16-APR-1986 & $.5 \mathrm{MeV}$ Neon & VDG & 4 Hrs. \\
\hline 17-APR-1986 & $.5 \mathrm{MeV}$ Neon & VDG & 4 Hrs. \\
\hline 5-MAY-1986 & $.9 \mathrm{MeV}$ Neon & VDG & $4 \mathrm{Hrs}$. \\
\hline 16-MAY-1986 & 1.4 MeV Nickel +1 & Tandem & $6 \mathrm{Hrs}$. \\
\hline 19-MAY-1986 & 1.4 MeV Nickel +1 & Tandem & $7 \mathrm{Hrs}$. \\
\hline 4-JUN-1986 & 1.3 MeV Neon & VDG & 4 Hrs. \\
\hline 11-JUN-1986 & $1.3 \mathrm{MeV}$ Aluminum & Tandem & $8 \mathrm{Hrs}$. \\
\hline 12-JUN-1986 & $1.3 \mathrm{MeV}$ Aluminum & Tandem & $4 \mathrm{Hrs}$. \\
\hline $16-J U N-1986$ & $1.3 \mathrm{MeV}$ Aluminum & Tandem & $8 \mathrm{Hrs}$. \\
\hline 17-JUN-1986 & $1.3 \mathrm{MeV}$ Aluminum & Tandem & $8 \mathrm{Hrs}$. \\
\hline 1-JUL-1986 & $1.3 \mathrm{MeV}$ Neon & VDG & $4 \mathrm{Hrs}$. \\
\hline 7-JUL-1986 & 1.4 MeV Chromium (Try) & Tandem & $4 \mathrm{Hrs}$. \\
\hline $8-J U L-1986$ & $1.4 \mathrm{MeV}$ Chromium & Tandem & $8 \mathrm{Hrs}$. \\
\hline 14-JUL-1986 & $1.4 \mathrm{MeV}$ Chromium & Tandem & $4 \mathrm{Hrs}$. \\
\hline 22-JUL-1986 & $5.0 \mathrm{MeV}$ Nickel +2 & Tandem & 8 Hrs. \\
\hline 23-JUL-1986 & $5,4,1,3 \mathrm{MeV}$ Nickel $+1,+2$ & Tandem & $7 \mathrm{Hrs}$. \\
\hline 24-JUL-1986 & 1.3 MeV Nickel +1 & Tandem & 4 \\
\hline
\end{tabular}


Air annealing ITO coated PLZT improves ITO sheet resistance by about 20\% at $150^{\circ} \mathrm{C}$, about $40 \%$ at $200^{\circ} \mathrm{C}$, and about $50 \%$ at $300^{\circ} \mathrm{C}$. Transmission either stays the same or increases about $1 \%$. Thus, it appears that thermal erasing

- of a voltage-cycled wafer will not harm the ITO and will actually improve it.

\section{PLZT OPTICAL TRANSMISSION MEASUREMENTS}

Transmission scans from 350 to $800 \mathrm{~nm}$ were run on most wafers at several key processing points: initial (new) condition, after implant, after anneal, and after ITO coating on both sides. Scans from 800 to $2800 \mathrm{~nm}$ were used on one sample from each implantation batch to characterize the implanted layer. Transmission measurements were made with a Beckman Instruments model 5270 spectrophotometer.

Transmission after implant was always lower than the new condition, with greatest differences in the 350 to $500 \mathrm{~nm}$ range and almost no difference about $800 \mathrm{~nm}$. The transmission spectra of implanted samples always displayed a low amplitude $( \pm 2 \%)$ oscillation due to the different refractive index of the implanted layer. The oscillation was easiest to discern and analyze at wavelengths from 1000 to $2000 \mathrm{~nm}$ (see Figure 9).

A 10 -hour anneal at $200^{\circ} \mathrm{C}$ in air always restored transmission to new condition levels and in some cases improved transmission in the 350 to $500 \mathrm{~nm}$ range over that of the new condition.

The impact on transmission of the ITO coatings is described in the previous section.

IMPLANTED PLZT REFRACTIVE INDEX, DENSITY, AND THICKNESS

After implantation of a PLZT wafer, an oscillation is observed in its transmission spectrum. The oscillation is due to the differing refractive indices of the implanted and unimplanted regions and results from constructive and destructive interference of light reflected from the air/layer and layer/wafer interfaces whenever quarter wavelength multiples of light equal the layer thickness.

The refractive index and thickness of the implanted surface layer on the PLZT wafer can be estimated quickly and reliably from spectrophotometer scans. 


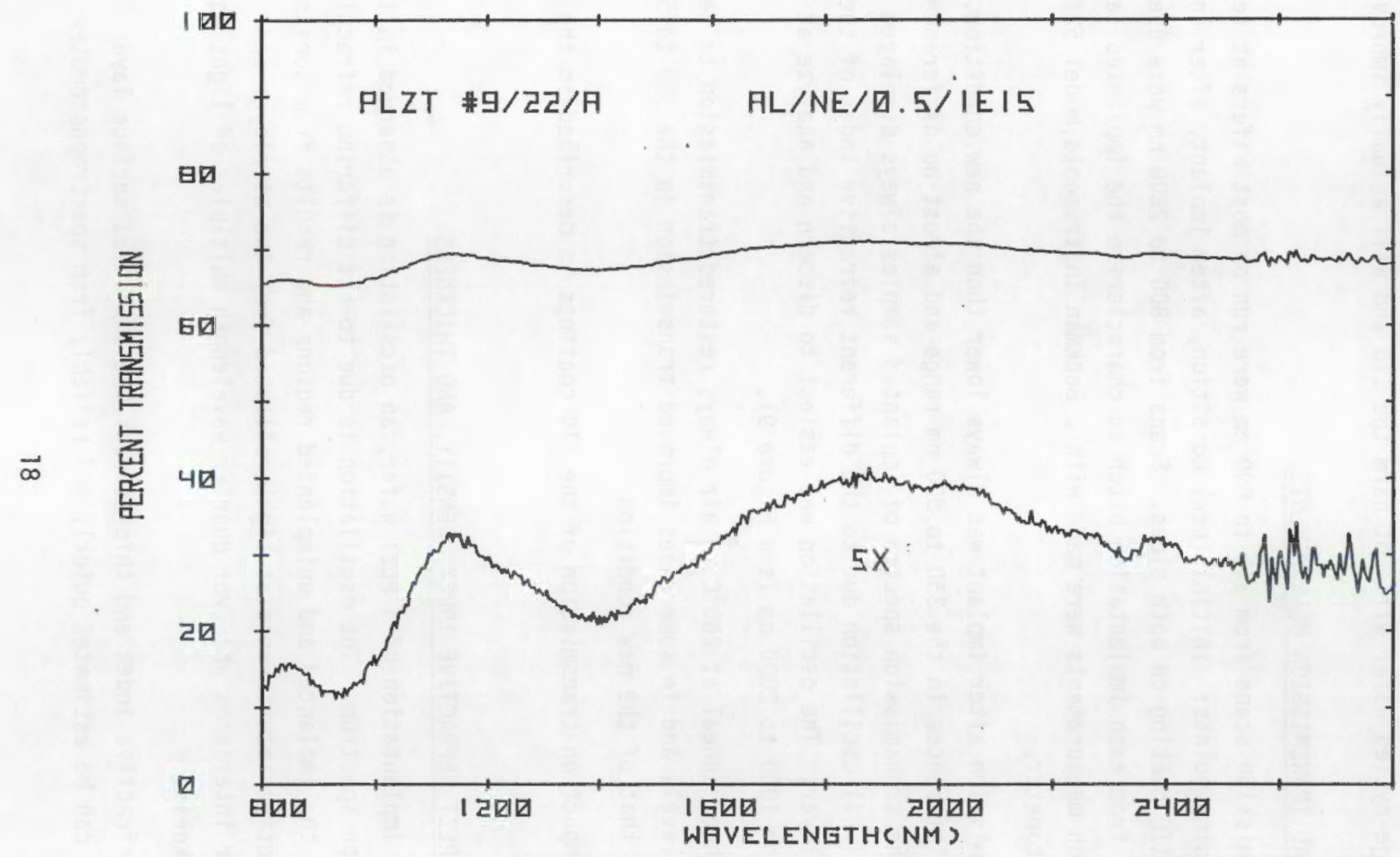

FIfiURE 9. IR Transmission 
The implanted layer is assumed to be altered uniformly in depth, and any of the many analysis techniques for a single layer optical coatings on a substrate of known optical properties can be used. With the envelope technique of Manifacier (Manifacier 1976), for example, the refractive index of the implanted layer can be calculated from simple equations using the maximum and minimum transmission values in the osctilatory spectrum (see Figure 9) plus the refractive index of the unimplanted PLZT (2.55 near $1000 \mathrm{~nm})$. Transmission spectra in the infrared region of 1000 to $2800 \mathrm{~nm}$ are best to use to avoid short wavelength dispersion and any transmission loss introduced by implantation.

Once the refractive index $\mathrm{n}$ is calculated, the depth or thickness $t$ of the implanted layer can be estimated by numbering the transmission spectrum extrema according to the relation:

$$
4 n t=m l_{m} \quad(m=1,2,3, \ldots)
$$

where $I_{m}$ is the wavelength of the extremum. The extremum numbering is simplified by remembering that transmission peaks are even integers when the layer has higher refractive index than the unimplanted PLZT and odd integers for the reverse situation.

The density of the implanted layer relative to unimplanted PLZT can also be estimated from the refractive index using the simple relation:

$$
\left.\% \text { density }=(n-1) / n_{0}-1\right)
$$

where $n_{0}$ is 2.55 at 1000 to $2000 \mathrm{~nm}$. More accurate estimates can be made using exact effective medium theory equations (Jacobsson, 1975).

In all wafers examined this year, wafer transmission was enhanced over unimplanted PLZT and maxima occurred at odd numbered wavelengths. Thus, the implanted layer was always lower in refractive index and density than unimplanted material. Representative values for some of the implants are listed in Table 3.

Implanted layer refractive indices range form 2.29 to 2.45 compared to unimplanted values of 2.55. These reduced indices correspond to densities of 83 to $94 \%$, suggesting that ion implantation introduces a considerable network of voids. 
TABLE 3 . PLZT IMPLANTED LAYER CHARACTERIZATION

\begin{tabular}{|c|c|c|c|c|c|}
\hline \multirow[b]{2}{*}{$P L Z T=$} & & \multicolumn{2}{|c|}{ REFRACTIYE (a) RELATIYE } & \multirow[b]{2}{*}{$\begin{array}{l}\text { THICKNESS } \\
\text { (MICROUS) }\end{array}$} & \multirow[b]{2}{*}{$\begin{array}{c}\operatorname{STRESS}^{(b)} \\
\left(10^{8}{ }^{\left.\text {DYMES } / \mathrm{CM}^{2}\right)}\right.\end{array}$} \\
\hline & IMPLANTS & $\begin{array}{r}\text { INDEX } \\
\text { (MIR) } \\
\end{array}$ & $\begin{array}{l}\text { DENSITY } \\
\text { ( } \mathrm{g}) \\
\end{array}$ & & \\
\hline $87(1)$ & $\begin{array}{l}\text { 1. } 4 \mathrm{MeY} \mathrm{O}^{+}\left(2 \times 10^{15}\right)+ \\
\text { 1.4 } \mathrm{MeY} \mathrm{Ni}^{+}\left(1 \times 10^{15}\right)^{+}\end{array}$ & 2.44 & 94 & 1.18 & $570(c)$ \\
\hline M5 & $\begin{array}{l}500 \mathrm{Ker} \mathrm{Me}^{+}\left(1 \times 10^{15}\right)+ \\
1.4 \mathrm{Mer}^{+}\left(2 \times 10^{15}\right)\end{array}$ & 2.45 & 94 & 1.14 & $612(c)$ \\
\hline $4 B$ & $500 \mathrm{KeY} \mathrm{Me}+\left(1 \times 10^{15}\right)$ & 2.29 & 83 & 0.67 & $250(C)$ \\
\hline $7 / 23 \mathrm{~A}$ & $\begin{array}{l}\text { 1.3 } \mathrm{MeY} \mathrm{Me}^{+}\left(2 \times 10^{15}\right)+ \\
\text { 1.4 } \mathrm{MeY} \mathrm{Cr}^{+}\left(8 \times 10^{14}\right)\end{array}$ & 2.45 & 93 & 0.89 & $22(C)$ \\
\hline $7 / 25 \mathrm{~B}$ & $\begin{array}{l}1.3 \mathrm{MeY} \mathrm{Ne}^{+}\left(2.4 \times 10^{15}\right) \\
1.3 \mathrm{MeY} \mathrm{Al}^{+}\left(1 \times 10^{15}\right)\end{array}$ & +2.43 & 92 & 1.20 & 18 (c) \\
\hline
\end{tabular}

(a) UMIMPLANTED WAFER IS 2.55

(b) $\mathrm{C}=$ COMPRESSIYE 
Thicknesses estimated from the interference patterns agree well with those estimated from stopping range calculations for energetic ions impinging on a solid. The optically determined depth is just beyond the maximum in the calculated damage versus depth curve.

\section{PLZT CURVATURE AND MECHANICAL STRESS}

PLZT wafer curvature was measured interferometrically using a Tropel Model 90001 grazing incidence laser interferometer with variable fringe sensitivity from 0.1 to 15 microns/fringe. A vacuum pen holds the fragile wafer and allows tilting as well as translation of the wafer toward and away from the optical flat to produce the most symmetric (and hence simplest to interpret) fringe pattern. Fringe spacing was calibrated before each measurement using a reference wedge traceable to NBS. Interferograms are displayed on a TV monitor and can be photographed for permanent records.

Curvature measurements can be used to deduce mechanical stress levels in a coating or implanted layer using the following relation (Hoffman; Glang):

$$
S=\left[E_{p} / 3\left(1-v_{p}\right)\right]\left[t_{p}{ }^{2} / t_{c} r_{p}{ }^{2}\right] d
$$

where $S$ is the stress in the coating or implanted layer

$E_{p}$ is Young's Modulus for PLZT $\left(6.3 \times 10^{11}\right.$ dynes $\left./ \mathrm{cm}^{2}\right)$

$v_{p}$ is Poisson's ratio for PLZT $(0.3)$

$t_{p}$ is the PLZT thickness

$t_{c}$ is the coating or implanted layer thickness

$r_{p}$ is the PLZT radius

and $d$ is the PLZT deformation (edge displacement versus center) due to stress in the coating or the implanted layer.

The same equation is also used later to estimate stress in the ITO coating when the deformation $d$ is caused by application of a voltage to the two ITO electrodes. A concave curvature of the coated or implanted side corresponds to a tensile stress in the coating, and a convex curvature to a compressive stress. Curvature can be determined by the inward or outward motion of the fringes when the substrate is moved away from the optical flat. The substrate 
deformation $d$ is measured by counting fringes and multiplying by the fringe sensitivity.

Curvature measurements were made as a function of processing step on selected wafers from several implantation batches. Processing steps examined include initial (new) condition, after dicing, after implant, after anneal, and after ITO coating application. Table 4 summarizes results for three wafers of one implantation batch. The results and those obtained for other batches of wafers lead to the following conclusions:

- 1-inch square new hot-pressed wafers have slight curvature before dicing. All are saddle-shaped, with curvatures ranging 0.25 to $3.3 \mathrm{per}$ quarter inch. The implant-introduced curvature mentioned in the next paragraph can be reduced by identifying and implanting the concave side of each wafer.

- Implants introduce a large curvature. The implanted side of the wafer is always convex, indicating a compressive stress in the implanted layer. The magnitude of the compressive stress is dependent on ion species, energy and implant flux. Values observed throughout the year ranged from 10 to 50 microns per quarter inch. Figure 10 is a sketch of what the implanted layer looks like if these curvature results are combined with the spectrophotometric results of the previous section.

- An ITO coating on the implanted face adds a small tensile stress which partially cancels the curvature introduced by the implanted layer, but it is not large enough to help much. The implanted layer compressive stress is typically 100 times larger than the ITO tensile stress. ITO coating the other side of the wafer cancels the effects of ITO on the first side coated so that the ITO has no net impact on wafer curvature.

- PLZT and ITO are well matched in thermal expansion coefficient (about $5 \times 10^{-6} / \mathrm{C}$ ), so that there is no temperature-dependent curvature of the wafer expected from the ITO coating.

A 10 -hour $/ 200^{\circ} \mathrm{C} /$ air anneal removes the very large and significant implantinduced curvature. Table 5 illustrates the beneficial effects with numbers and interferograms. Curvature (stress) is reduced to the level of new wafers. The 


\section{TABLE 4}

PlzT CuRVature(A) (MEChanical STRESS) vs. PROCESSING STEP

CASE: $10^{15} / \mathrm{cm}^{2} 500 \mathrm{KeV} \mathrm{Ne}^{+}$plus $1-1.7 \times 10^{15} / \mathrm{cm}^{2} 1.4 \mathrm{MeV} \mathrm{Ni}^{+}$

\begin{tabular}{|c|c|c|c|c|}
\hline $\begin{array}{c}\text { WAFER } \\
\quad= \\
\end{array}$ & INITIAL (C) & $\begin{array}{l}\text { AFTER }^{(D)} \\
\text { IMPLANT }\end{array}$ & $\begin{array}{l}\text { AFTER ITO(E) } \\
\text { ON IMPLANI }\end{array}$ & $\begin{array}{l}\text { AFTER ITO(F) } \\
\text { ON OTHER SIDE }\end{array}$ \\
\hline$c-13(1)$ & +1.3 & -10.2 & -9.4 & -10.9 \\
\hline$c-13(2)$ & +1.2 & -10.4 & -10.2 & -10.2 \\
\hline$c-13(3)$ & -1.5 & -10.8 & -10.1 & -10.8 \\
\hline
\end{tabular}

(A) Microns deflection edge vs. center. - implanted side convex, + concave.

(B) $0.5 \times 0.5$ inch, 90/65 PLZT.

(C) All are identical. Side selection arbitrary at this point.

(D) May vary with implant species, density, and energy.

(E) Expected ITO contribution is +0.2 .

(F) Coating both sides with same thickness produces no net change in curvature. 


\section{FIGURE 10 \\ PLZT WAFER AFTER IMPLANT}

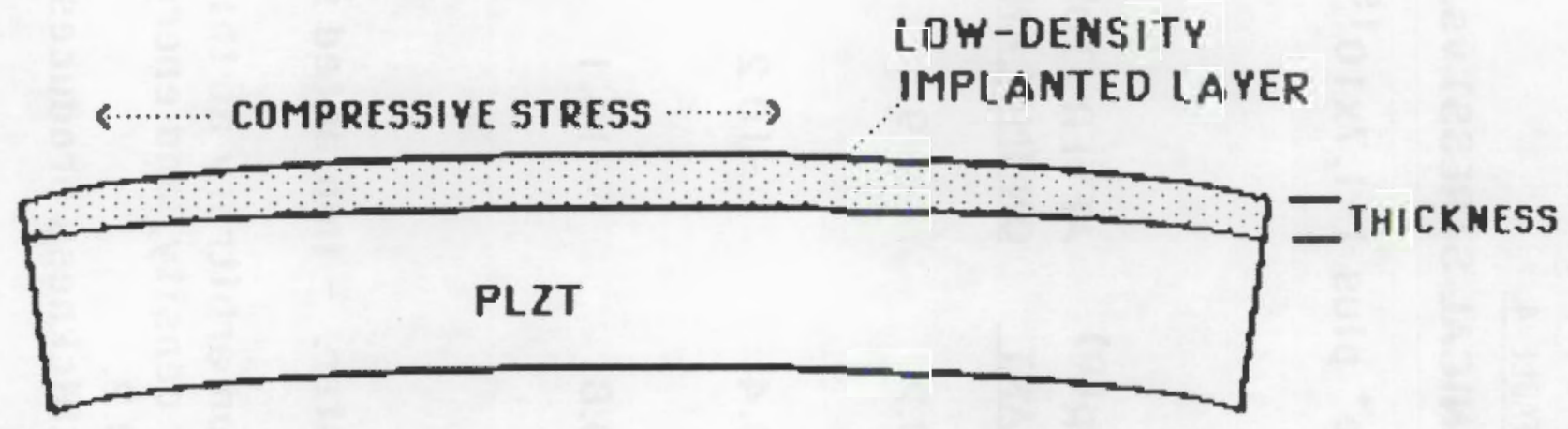


TABLE 5. Reduction of Implant-Induced Wafer Curvature by $200^{\circ} \mathrm{C} / 10$ hour / Air Anneal

\begin{tabular}{lllllll} 
WAFER * & STEP & $C_{x}$ & $C_{-x}$ & $C_{y}$ & $C_{-y}$ & $C_{\text {AVE. }}$ \\
9 & & & & & & \\
$9 / 13 \mathrm{~A}$ & BEFORE & -6.66 & -5.33 & -30.0 & -18.6 & -15.1 \\
& AFTER & +2.80 & +3.20 & +3.20 & +3.12 & +3.08 \\
\multirow{2}{*}{$9 / 14 B$} & BEFORE & -3.39 & -1.70 & -27.1 & -20.3 & -13.1 \\
& AFTER & +4.16 & +6.00 & +4.00 & +4.80 & +4.74
\end{tabular}

CURVATURES IN MICRONS/0.25 INCH

- MEANS IMPLANT SIDE CONYEX

+ MEANS IMPLANT SIDE CONCAVE

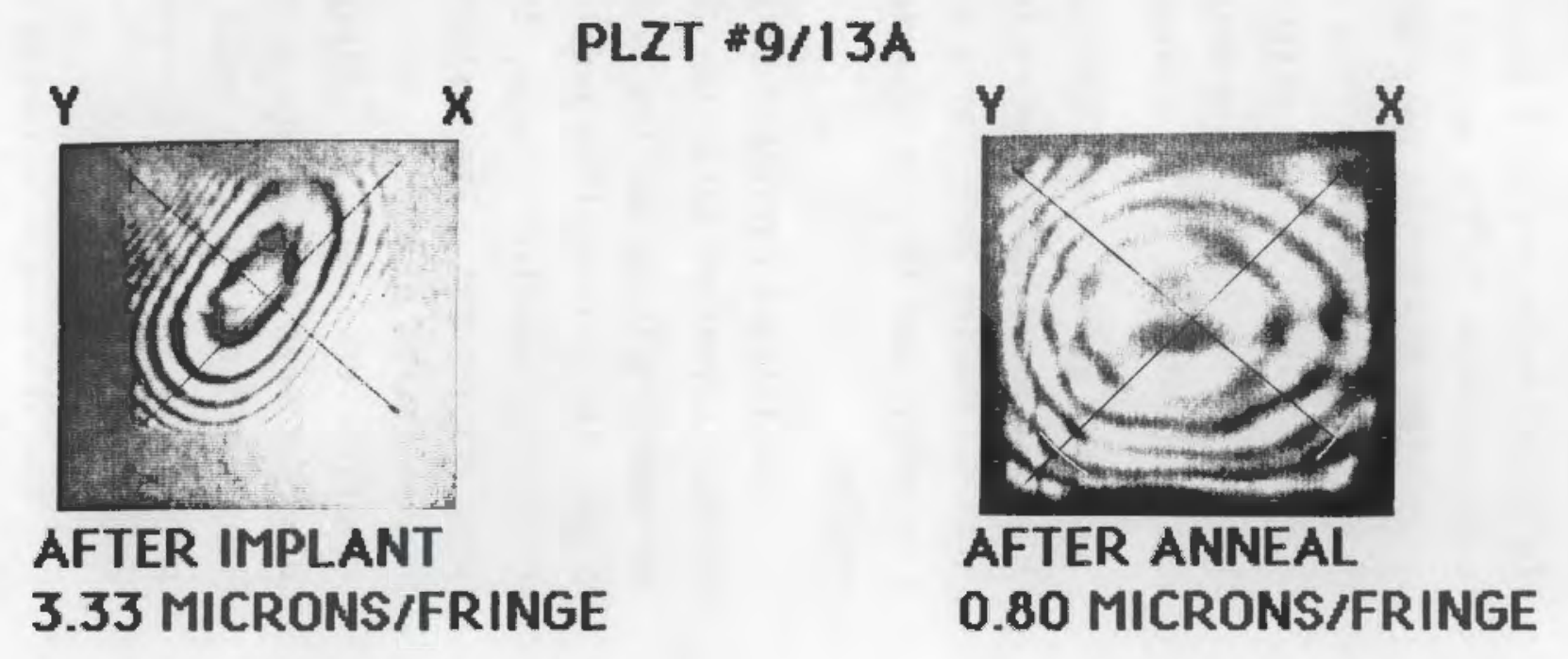


implementation of the annealing step into the wafer processing sequence is highly recommended.

\section{PLZT CURVATURE VERSUS APPLIED VOLTAGE}

The interferometer described above was adapted for measurement of wafer deformation when a cyclic voltage $( \pm 400 \mathrm{~V})$ is applied to the ITO coatings. The ITO coated wafer was held by the center of one face with the vacuum pen, close to and parallel to the optical flat. One lead of a dc voltage supply was attached to the vacuum pen, which in turn contacted the ITO coating on one side of the wafer. The other lead was attached to a thin flexible lead cut from copper adhesive tape and attached to the corner of the ITO coating on the other side of the wafer. When a voltage is applied, the wafer distortion is immediately recorded in the displayed interferogram.

Three wafers from different implant batches were examined this year. Figure 11 shows the recorded interferograms and the measured curvatures (microns/quarter inch) for a wafer cycled from 0 to $+400 \mathrm{~V}$, back to $0 \mathrm{~V}$, from 0 to $-400 \mathrm{~V}$, and finally back to $0 \mathrm{~V}$ again. Two other wafers showed similar results.

The initial curvature is about 4 microns/quarter inch (convex), the residual curvature after the 10 hour $/ 200^{\circ} \mathrm{C} / \mathrm{air}$ anneal. The wafer flattens with increasing voltage applied to the implanted side as the ferroelectric domains align. The polarization remains when the voltage is reduced to zero. With increasing negative voltage, the polarization is cancelled, and the convex curvature returns. The hysteresis behavior associated with polarization and depolarization of the ferroelectric material thus manifests itself in the curvature versus applied voltage curve. Instead of returning to the starting curvature when the voltage is reduced from $-400 \mathrm{~V}$ to $0 \mathrm{~V}$, however, an abnormal flip/flop occurs at high negative voltages which abruptly changes the wafer curvature. In at least one case, the curvature abruptly changed from convex to concave.

The stresses experienced by the ITO coatings as the wafer changes curvature $(\Delta d)$ with applied voltage can be calculated using the equation in the previous section. Figure 12 shows the results for the ITO-coated wafer of Figure 11. This particular wafer was annealed after the ITO coatings were 


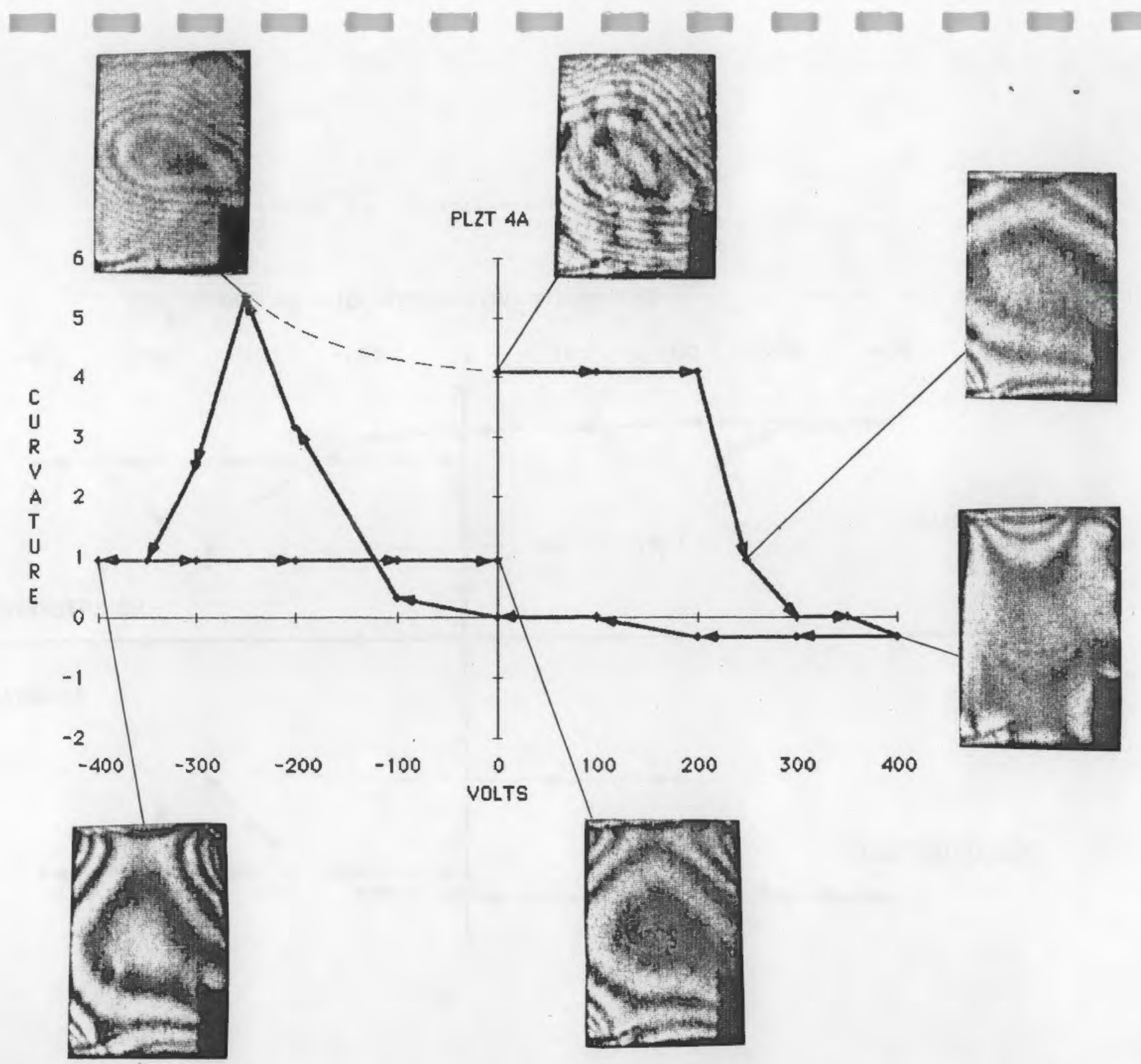

FIGURE 11. Implanted Sample Curvature Hysteresis 


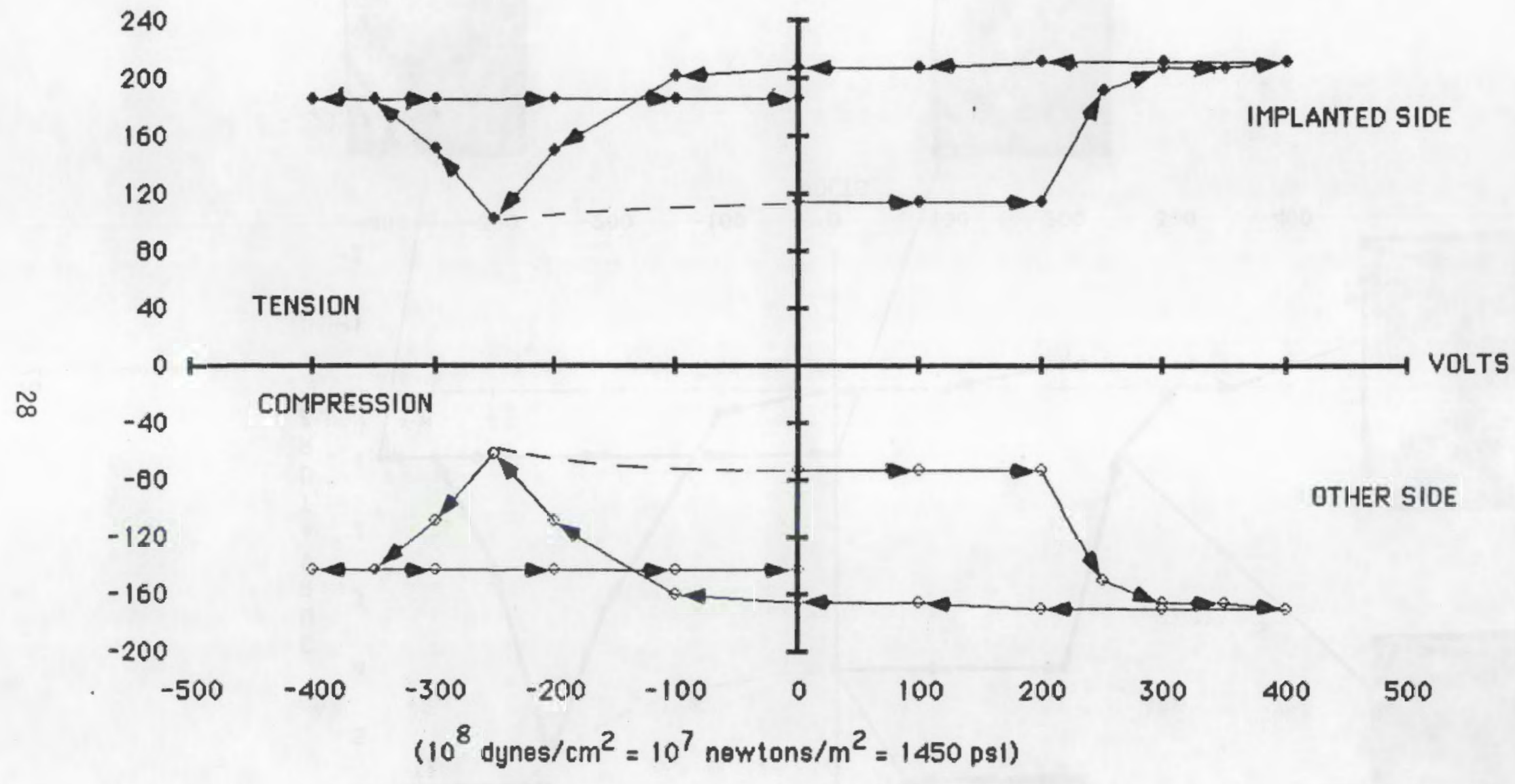

FIGURE 12. ITO Stress $\left(10^{8}\right.$ dynes $\left./ \mathrm{cm}^{2}\right)$ 
applied. Thus, the ITO coatings experience a stress of about $200 \times 10^{8}$ dynes $/ \mathrm{cm}^{2}$, even before the voltage is applied, due to anneal-induced wafer curvature change. Note that when one coating is put into compression, the other is put into tension. The curves are not exactly symmetric about zero stress because of the $20 \times 10^{8}$ dynes $/ \mathrm{cm}^{2}$ (tension intrinsic stress in the ITO coating). As the voltage is cyclically varied, the ITO coatings experience varying levels of compressive and tensile stresses.

The following important conclusions were learned from these curvature versus voltage measurements:

- The voltage dependence of PLZT curvature almost entirely controls ITO stress when on the normal hysteresis loop. ITO stress can be minimized only by minimizing the + and - voltages used.

- Implantation-induced stress does not affect ITO stress during the normal hysteresis loop. However, it may be responsible for the abnormal and potentially catastrophic mechanical flip/flop which occurs at high. voltages.

- ITO coatings on opposite PLZT sides experience almost equal stresses of opposite sign.

- ITO in compression will buckle or lift off if the PLZT/ITO adhesion is exceeded.

- ITO in tension will crack and pull apart if the ITO tensile strength is exceeded.

- No ITO failures were observed throughout the year. This means ITO/PLZT adhesion is good, and tensile stresses do not exceed the tensile strength of ITO.

In future work, the origin of the abnormal hysteresis should be further investigated by examining uniformly implanted PLZT, uniformly ITO-coated wafers, and unimplanted PLZT. 
PRELIMINARY THIN-FILM PLZT FABRICATION EXPERIMENTS

Six preliminary PLZT experiments were conducted to assess the level of difficulty and to determine the principal issues governing successful fabrication of thin-film PLZT with adaptive optics device potential.

The experiments were carried out in an rf diode sputtering system with a 4-inch diameter Motorola 9065 hot-pressed wafer target, argon and oxygen sputtering gases, fused silica and sapphire substrates, and a temperature-variable $\left(20\right.$ to $400^{\circ} \mathrm{C}$ ) substrate heater platform. Experiments were guided by important previous experimental results gleaned from a literature review of ten papers published by other workers.

Thin films made were characterized spectrophotometrically (thickness, refractive index, and qualitative assessment of stoichiometry) by x-ray diffraction (crystallinity, grain size, preferred orientation, and phase), and $x$-ray energy spectroscopy (absolute composition and comparison with starting target material).

The PNL preliminary experiments were very productive and resulted in the following conclusions:

- RF diode sputtering of PLZT is straightforward; no technical difficulties were encountered in fabricating thin films.

- Deposition rates are very good, $85 \mathrm{~A} / \mathrm{min}$ or $1 \mathrm{micron} / 2$ hours for medium target power levels.

- Oxidation appears completed based on visible transmission edge measured spectrophotometrically. Examples for a thin ( 0.355 microns) and a thick film (1.76 microns) are shown in Figure 13.

- Refractive index is low, 1.92 at $1000 \mathrm{~nm}$ compared to 2.55 for the starting Motorola 9065 hot-pressed wafer.

- Low temperature (about $200^{\circ} \mathrm{C}$ ) thin films are smooth and glass-like in appearance.

- High temperature (about $500^{\circ} \mathrm{C}$ ) thin films scatter noticeably.

- Both low and high temperature thin films are x-ray diffraction amorphous, although some sign of emerging crystallization is apparent for 


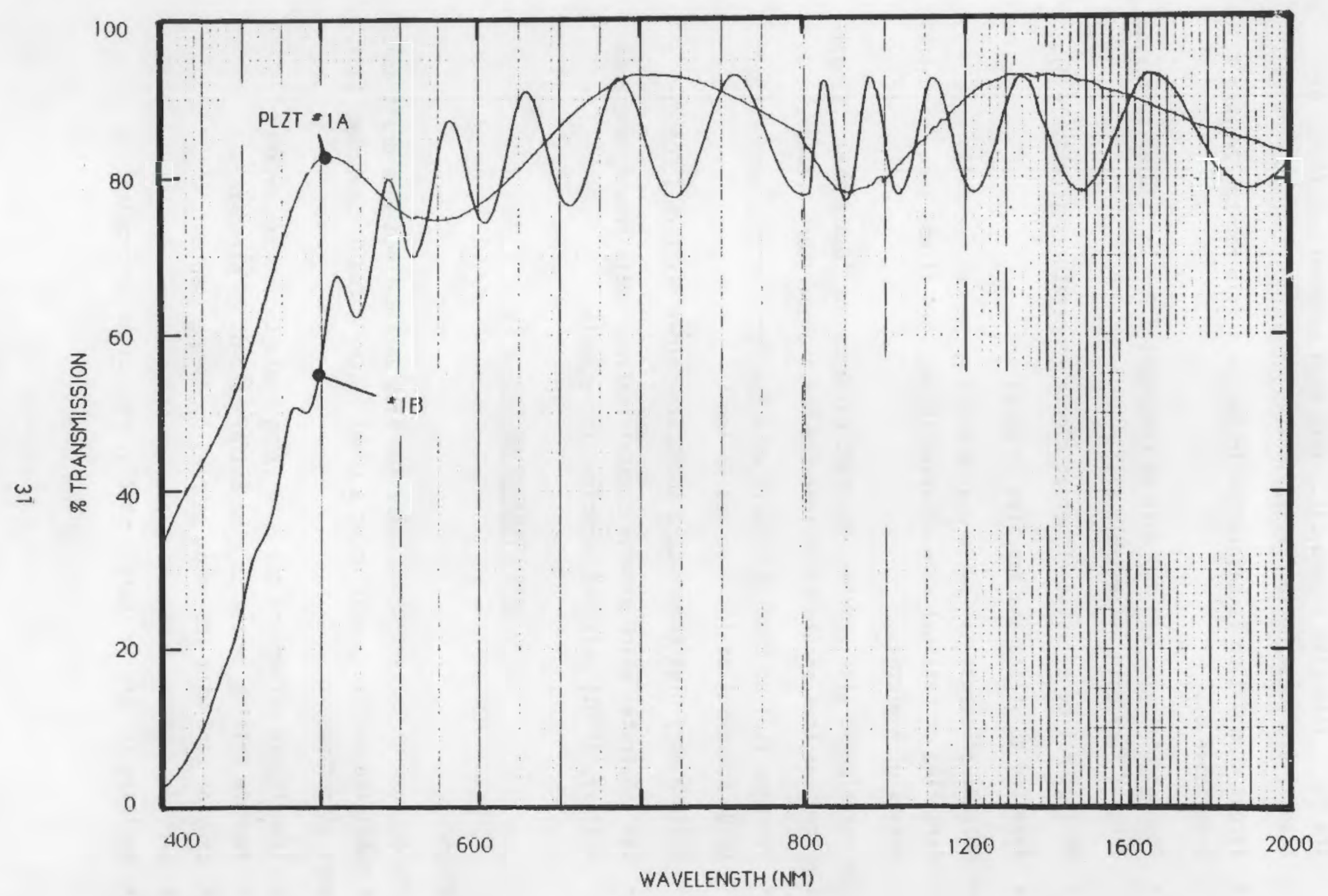

FIGURE 13. Thin Film PLZT Transmission 
the $500^{\circ} \mathrm{C}$ film (see Figure 14). Very high temperatures $\left(600^{\circ} \mathrm{C}\right.$ or higher) are required for crystallinity.

- Target composition is maintained in the thin film for $L a, Z r$, and $T i$ (see Table 6).

- $\mathrm{Pb}$ content is reduced and must be compensated for in future sputtering targets fabricated (see Table 6).

- $\mathrm{Pb}$ loss may be a little higher at higher temperatures (see Table 6).

- Adhesion to silica and sapphire is excellent.

- Mechanical stress is low to medium since no mechanical failures (cracking or peeling) were observed in any thin films, even for thicknesses of 4 microns.

The experiments also provide important guidance for future work. Experimental procedures that should be implemented for the next work include:

- Purchase PLZT sputtering targets with the ferroelectric composition (plus compensation for expected Pb loss).

- Build a very high temperature substrate holder platform $\left(>600^{\circ} \mathrm{C}\right)$.

- Use substrates which promote crystallization, grain growth, and even epitaxy, (0001) oriented sapphire, for example.

\section{CHARACTERIZATION TASK}

\section{OBJECTIVES}

The Optical Characterization tasks for FYB6 were defined in a draft annual report submitted to the sponsor under a cover letter dated 24 September 1986. They were as follows:

- investigate methods of characterizing implantation parameters

- improve writing system to give better control of parameters

- provide Land with implanted samples for comparison

- provide a means of measuring quantum efficiency

- evaluate and try to improve spatial resolution of samples 
FILENAME : WF 1C.RD

EILEHAHES:WP1F:RO
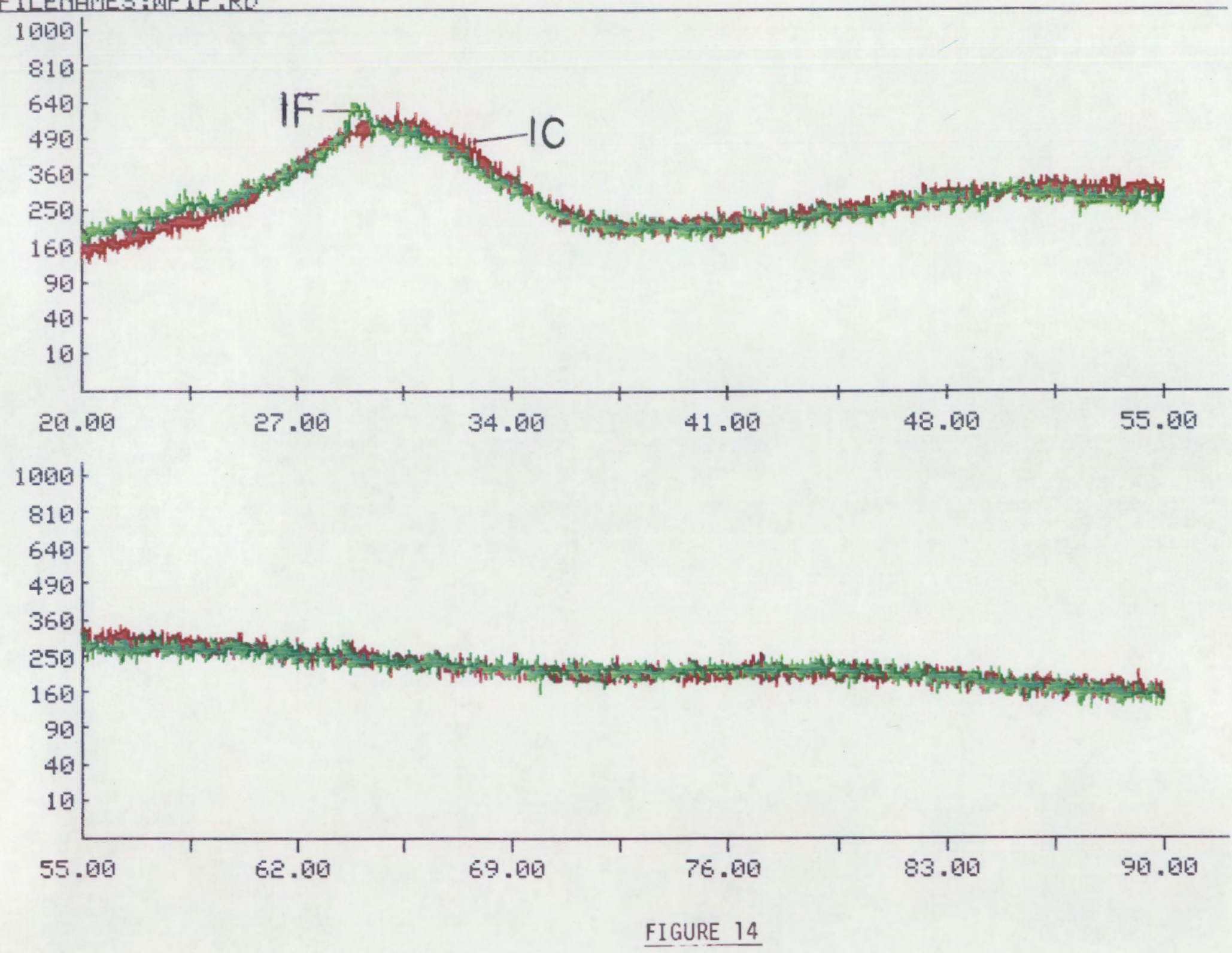

Thin Film PLZT K-ray Diffraction 
TABLE 6. XES COMPOSITIONAL ANALYSIS OF 9065 PLZT TARGET AND FILMS

(ATOM\%)

\begin{tabular}{lcccc}
\hline & $\underline{\mathrm{Pb}}$ & $\underline{\mathrm{La}}$ & $\underline{\mathrm{Zr}}$ & $\underline{\mathrm{Ti}}$ \\
TARGET (Nominal) & 90 & 10 & 65 & 35 \\
TARGET (Measured) & 89.6 & 10.4 & 64.1 & 35.9 \\
FILM $*$ IC (200 C) & 84.0 & 16.0 & 65.3 & 34.7 \\
FILM $=$ IF (500 C) & 82.3 & 17.7 & 67.5 & 32.5 \\
\hline
\end{tabular}




\section{CAPABILITY IMPROVEMENTS}

Early in the year, a number of characterization techniques were investigated. One worth mentioning was the use of an interferometer to try to evaluate the surface deformation due of a stored hologram as a means of determining photosensitivity. Several samples were exposed and examined using a MachZehnder interferometer. A typical sample interferogram pair is shown in Figure 15. Surface deformation is sufficient to make precise measurements of photosensitivity, but was not explored further due to other more pressing priorities and the need for a means of quantitatively evaluating the interferograms. Commercial hardware and software is available to implement the measurement, but the value of such a measurement would need to be evaluated before the expense could be justified.

One action item remaining from the end of FY85 was to install a computercontrollable bipolar high voltage power supply. At the end of FY85, it had been ordered, but not yet delivered. Delivery was finally made in late August, 1986, and installation was postponed until early FY87 due to other priorities for september 1986, and dwindling funds. The installation of the power supply will complete automatic cycling of the bias voltage for the optical characterization system and eliminate the need for manual switching during the cycle.

Early in the year, the sponsor modified his software for biasing the samples during write cycles. The changes were incorporated into PNL's system.

A motor-driven sample holder was assembled for use in scanning samples. This may be used to implement measurements of uniformity of the implantations, but certainly will implement measurement of MTF. While these measurements were not attempted during FY86, they will be a priority for FY87.

The interferometer to be used in writing holograms to the samples was assembled, but changing priorities resulted in its not being used for any significant work during the year. Samples continued to be characterized primarily by exposing them to a relatively uniform beam from either a krypton ion laser or an arc lamp and tracing the hysteresis curve for photosensitivity data. The interferometer is planned for use in measuring sample MTF.

Some preliminary experiments were done on measuring diffraction efficiency using a neon/nickel coimplant, sample number 9/18B [previously numbered $\mathrm{C} 13(2)$ ]. 


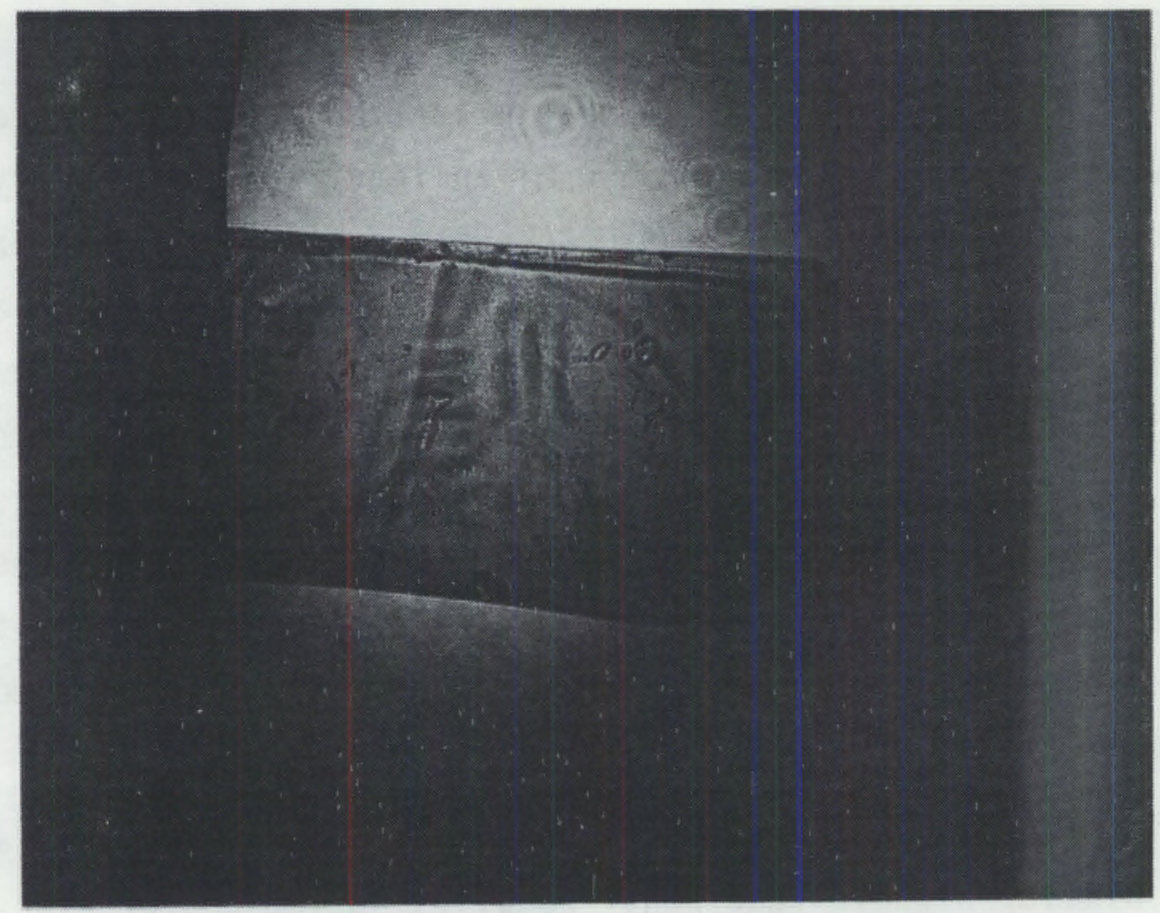

FIGURE 15a. Sample Photographed in Transmission

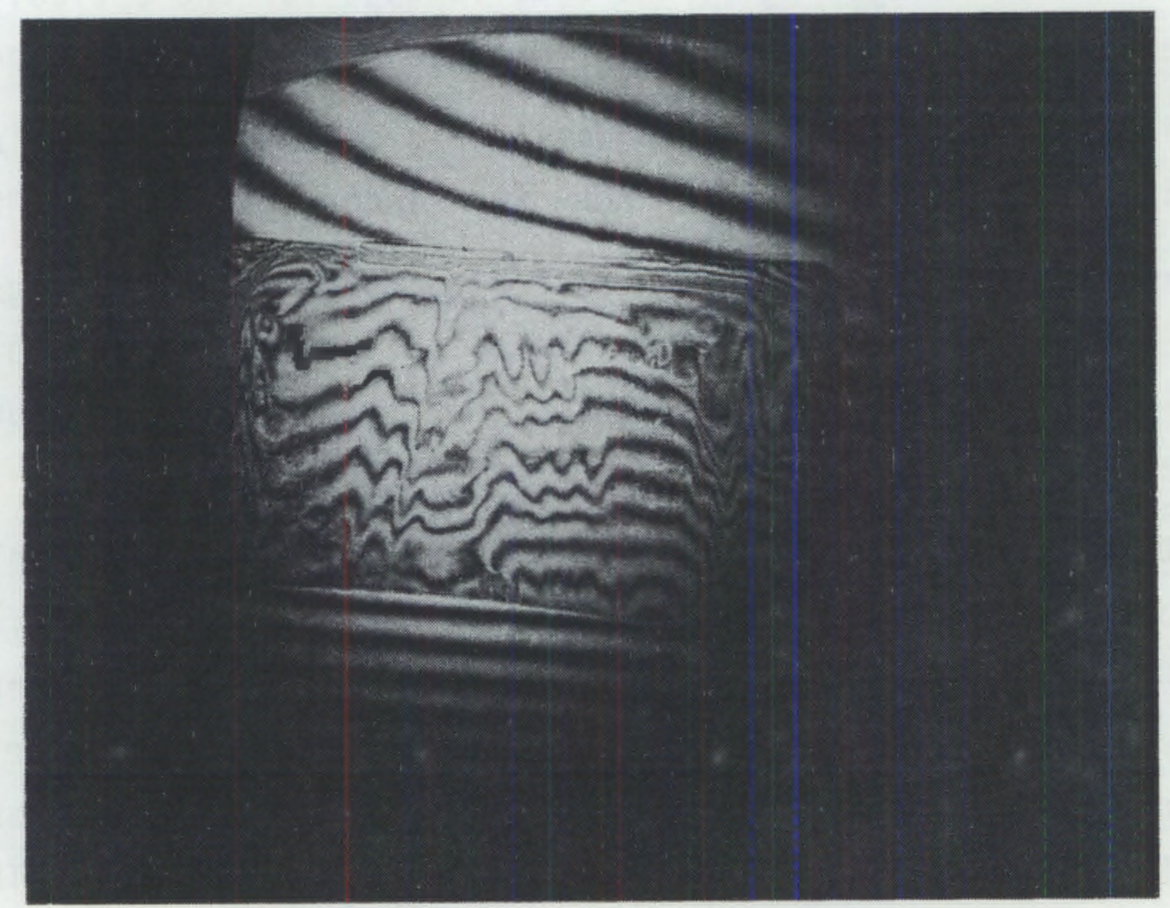

FIGURE 15b. Interferogram 
A 300 line/inch Ronchi grating was contact printed to the sample using a 90 $\mathrm{mW}$ exposure at $468 \mathrm{~nm}$ to 200 volts and $25 \%$ integrator voltage. This gave a faint image which when used to diffract a $3 \mathrm{~mW}$ HeNe beam resulted in the diffraction pattern shown in Figure 16. The first diffraction peak is at approximately $1 \mathrm{~cm}$ at a distance of $104 \mathrm{~cm}$ giving approximately $10 \mathrm{mR}$. The second order was not visible, and as seen in the Figure, was not recorded. In subsequent similar tests, comparable results were obtained. In one instance, measurements were made of the relative optical power giving approximately $3 \%$ to $5 \%$. In another test, the sample was lightly coated with aluminum to enhance reflection and the relative optical powers were $1.5 \%$ to $1.8 \%$ on reflection compared to $2.2 \%$ to $2.3 \%$ for transmission through the uncoated sample.

In a third test, using $\mathrm{Ne} / 0$ implanted sample $9 / 19 \mathrm{~B}, 30 \mathrm{~mW} / \mathrm{sq}-\mathrm{cm}$ at $468 \mathrm{~nm}$, ramped to 200 volts and allowed to expose for 2 minutes, the center of the sample was probably overexposed, but the outer portions recorded the 300 line/inch Ronchi grating. Figure 17 shows the diffraction pattern using a 25 $\mathrm{mW}$ HeNe laser. The second order diffraction peaks are clearly visible. Previous samples were rechecked with the $25 \mathrm{~mW}$ laser and the second orders were still not visible. The relative power of the orders was not measured with this sample.

\section{TRANSMISSION MEASUREMENTS}

A series of transmission measurements of substrates was done to determine the uniformity of the raw material and the materials at various processing steps. These were for $70 / 65$ samples provided by the sponsor. Selected transmission plots are shown in Figures 18 through 21 which reflect expected 70/65 transmission cutting off at about $400 \mathrm{~nm}$ and relatively flat at about $70 \%$. Implanted samples show a $90 \%$ transmission peak at about $1100 \mathrm{~nm}$ to $1200 \mathrm{~nm}$, and a gradual falloff to about $40 \%$ to $50 \%$ at $2500 \mathrm{~nm}$. For comparison, Figure 22 shows the transmission of the 70/30 raw material provided by the sponsor in May.

In January, a sample of $7 / 65 / 35$ PLZT was implanted with oxygen ions in a test to determine if photosensitivity was enhanced. At that time, PNL was not prepared to make measurements of the type being made by Land. A single onecentimeter square sample was produced and sent to the sponsor who had Land 


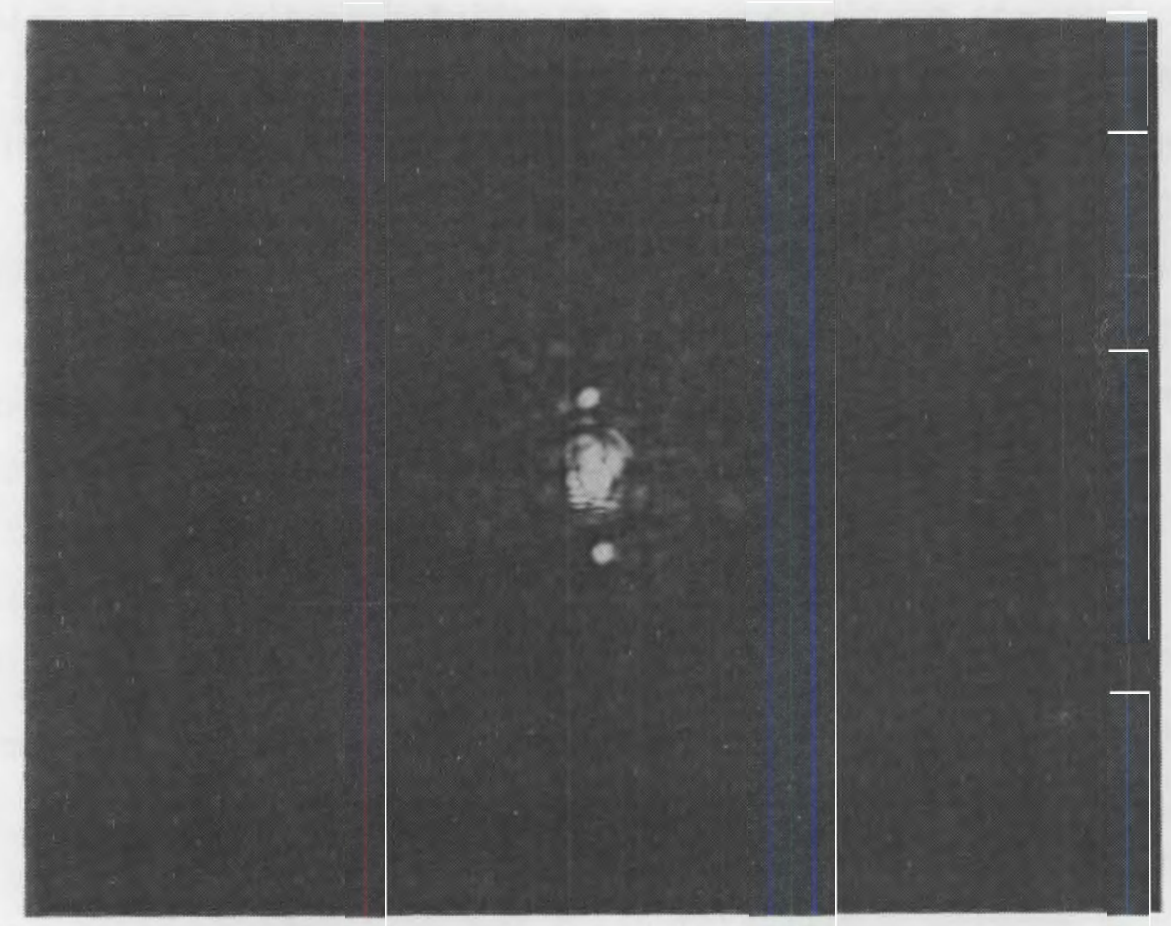

FIGURE 16. Diffraction of $3 \mathrm{mll}$ HeNe Beam by 300 Line/Inch Ronchi Ruling Printed on Sample 9/18B

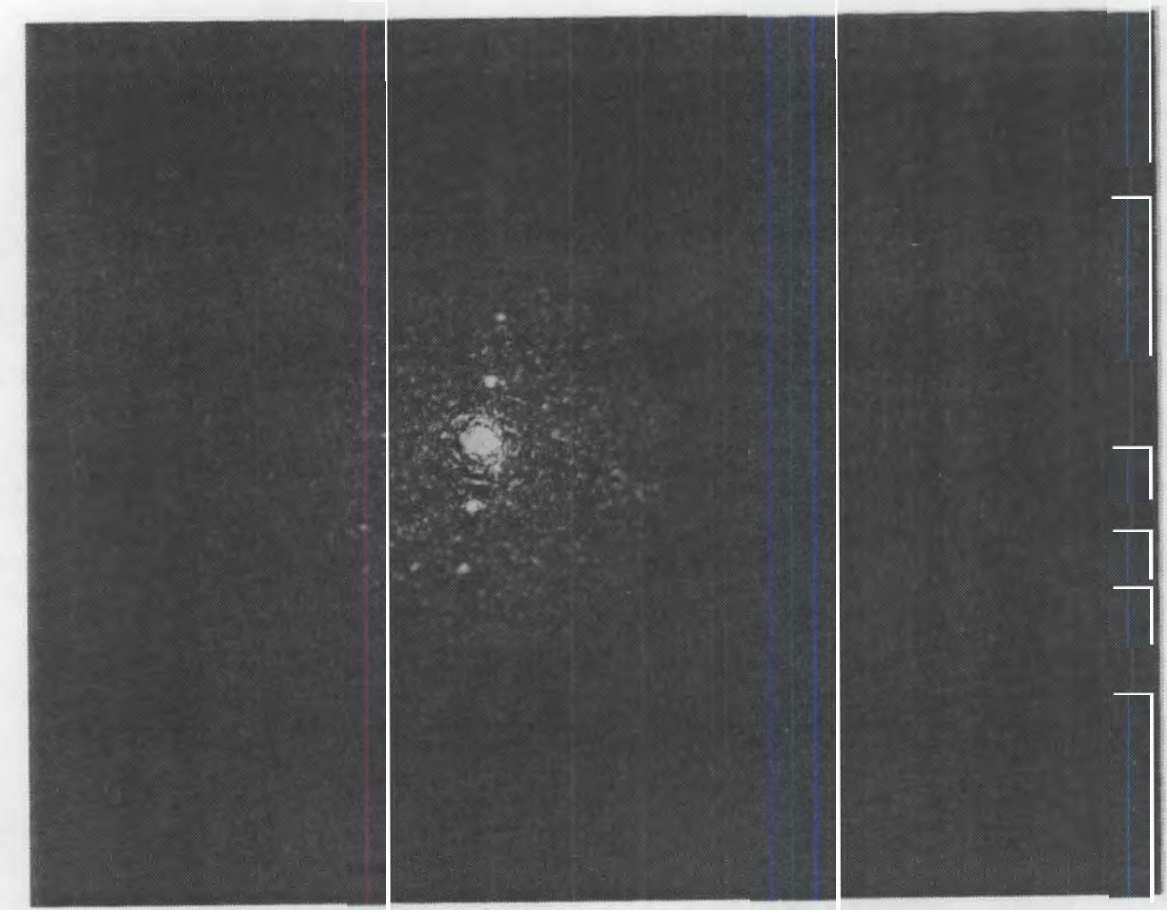

FIGURE 17. Diffraction of $25 \mathrm{mll}$ HeNe Beam by 300 Line/Inch Ronchi Ruling Printed on Sample 9/19B 


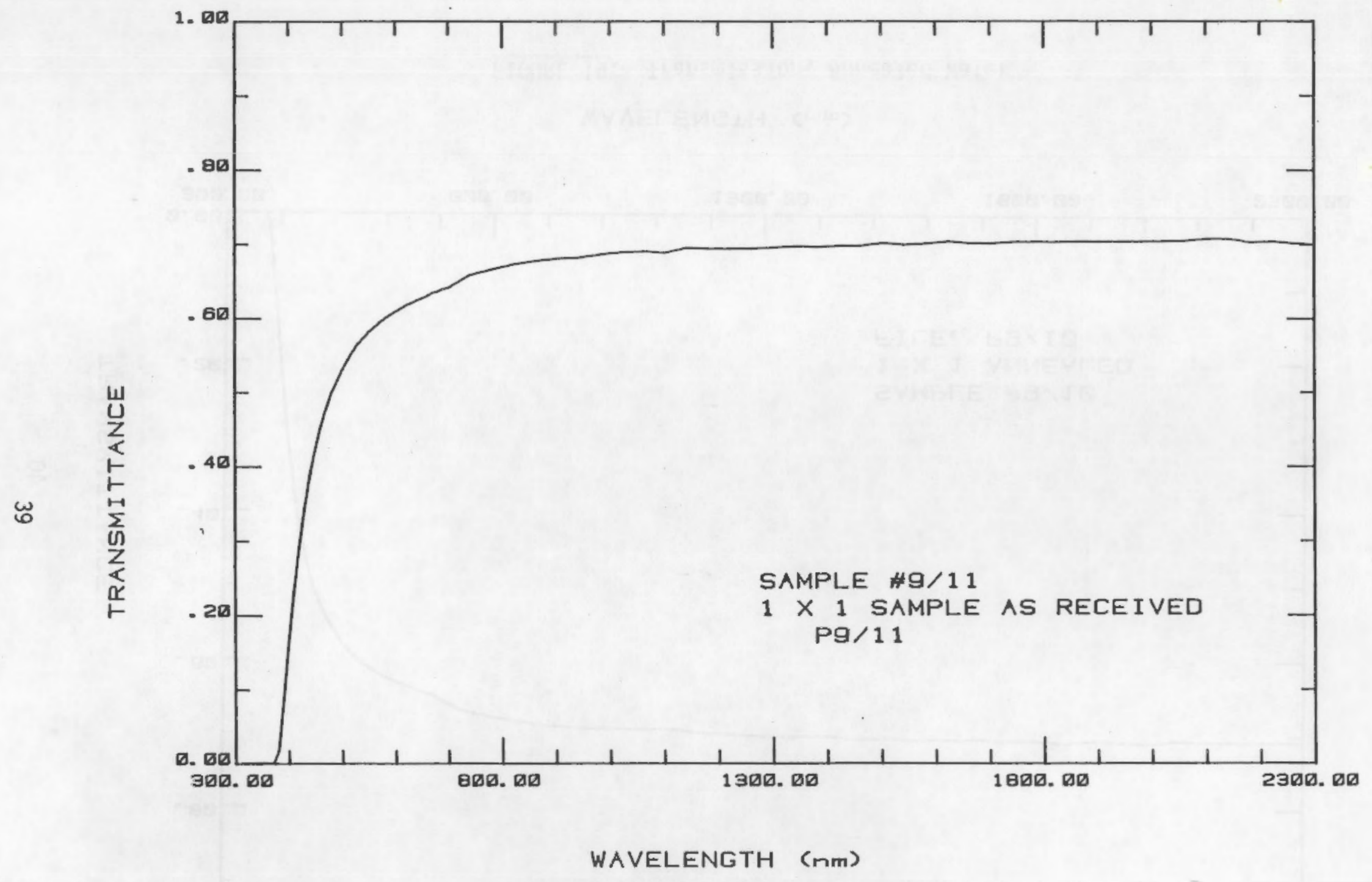

FIGURE 18. Transmission, As-Received Wafer 


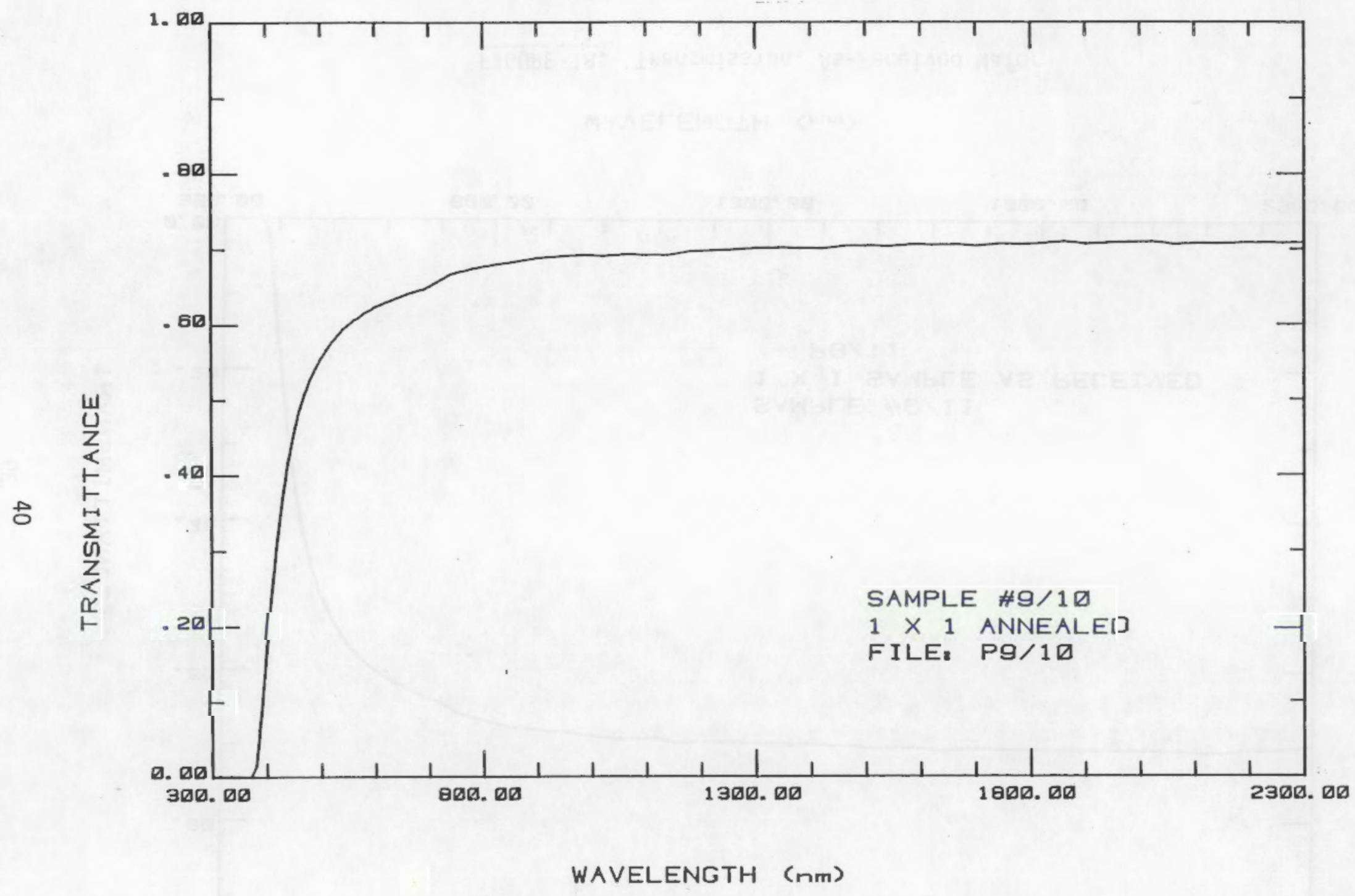

FIGURE 19. Transmission, Annealed Wafer 


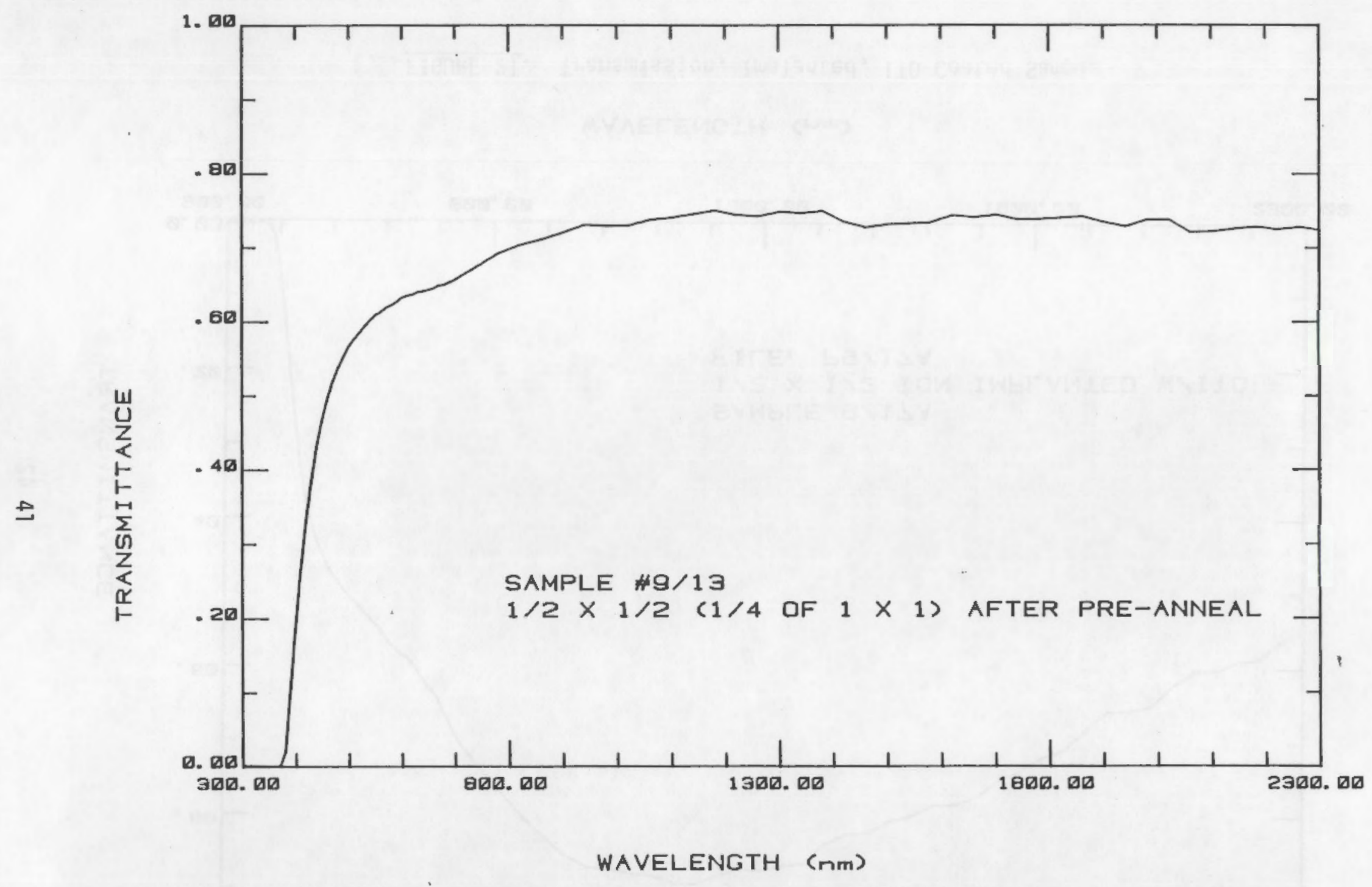

FIGURE 20. Transmission, Cut and Annealed Wafer 


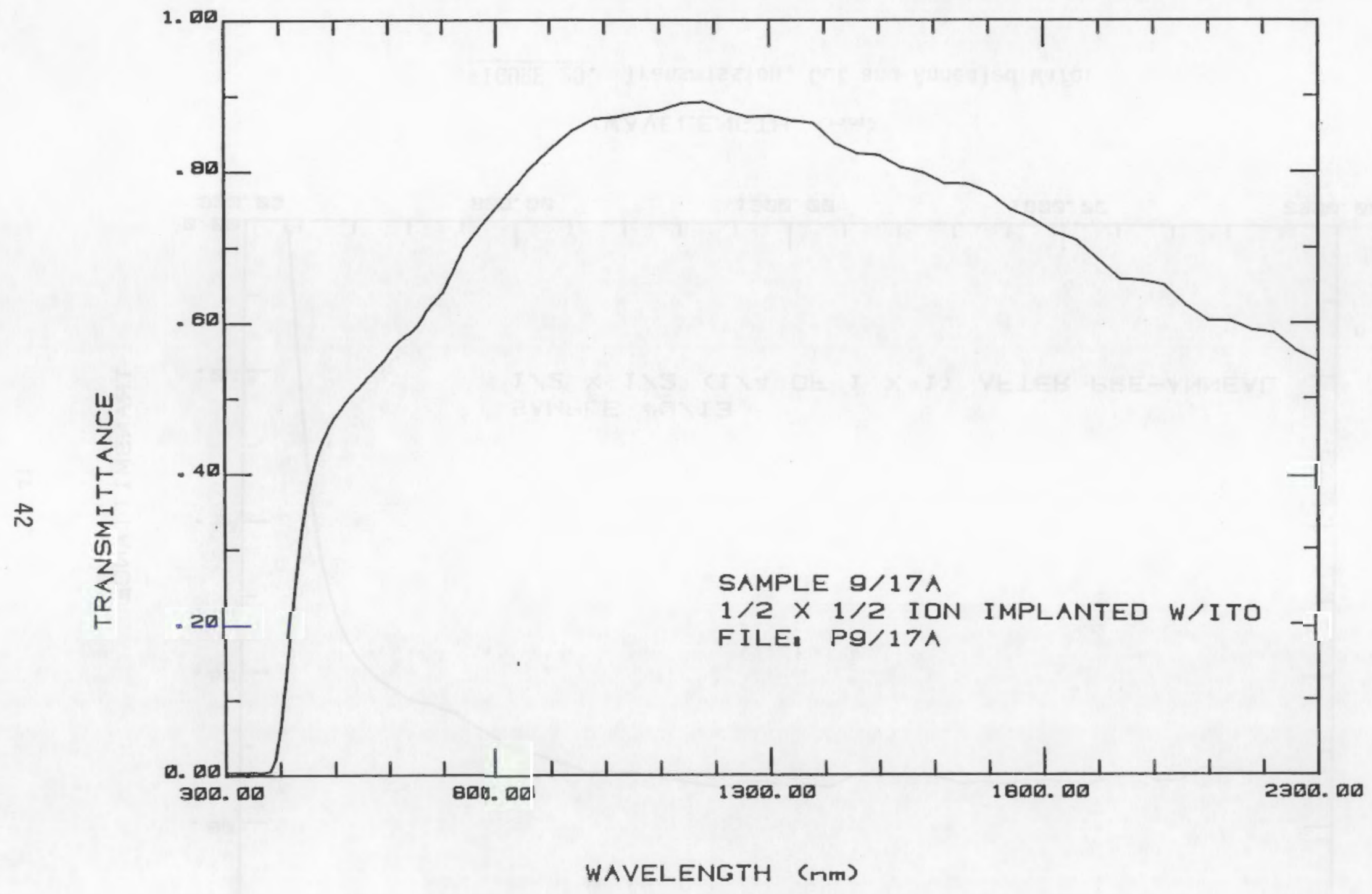

FIGURE 21. Transmission, Implanted, ITO Coated Sample 


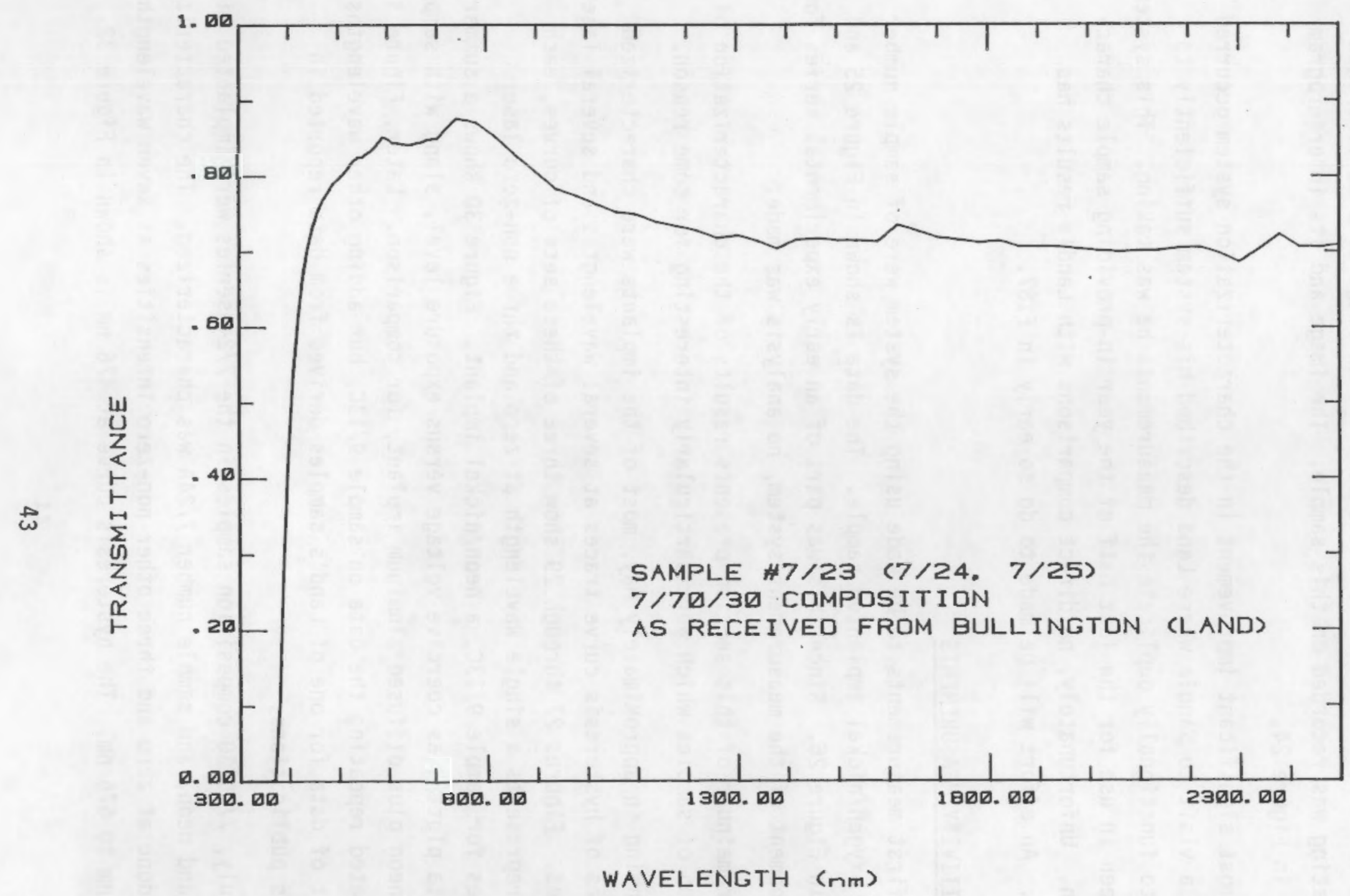

FIGURE 22. Transmission, As-Received 7/70/30 Wafer 
measure it. The results were that it showed not only an enhanced photosensitivity, but also a flatter spectral response. The plot shown in Figure 23 is a characteristic curve of the implanted sample. An image of a 50 line/inch Ronchi grating was recorded on this sample. The image and its interferogram are shown in Figure 24.

The most significant improvement in the characterization system occurred following a visit to Sandia where Land described his system sufficiently to allow us to functionally duplicate the measurements he was taking. This system has now been in use for the last half of the year in providing sample characterization. Unfortunately, no direct comparisons with Land's results has been made. An effort will be made to do so early in FY87.

\section{PHOTOSENSITIVITY MEASUREMENTS}

The first measurements to be made using the system were of sample number $9 / 17 \mathrm{C}$, an oxygen/nickel implanted sample. The data is shown in Figure 25 and expanded in Figure 26. Since this was part of an early experimental series for the development of the measurement system, no analysis was made.

The remainder of this section presents results of the characterization of a selection of samples which were particularly interesting for some reason.

Beginning in approximately May, most of the implants were characterized by a series of hysteresis curve traces at several wavelengths and several laser intensities. Figures 27 through 29 show three of these sets of curves, each of which represents a single wavelength at zero and three non-zero laser intensities for sample $9 / 13 \mathrm{C}$, a neon/nickel implant. Figure 30 shows a summary of the data plotted as coercive voltage versus exposure level, along with sample $9 / 22 B$, a neon plus diffused aluminum implant, for comparison. Later, Figure 31 was generated repeating the data on sample $9 / 13 \mathrm{C}$, but adding other wavelengths and a plot of data for one of Land's samples derived from data reported in one of his publications.

In July, $7 / 70 / 30$ composition samples in the $7 / 24$ series were implanted with aluminum and neon, and sample number 7/24A was characterized. The characterization was done at zero and three other non-zero intensities at seven wavelengths from $476 \mathrm{~nm}$ to $676 \mathrm{~nm}$. The hysteresis curve at $476 \mathrm{~nm}$ is shown in Figure 32 


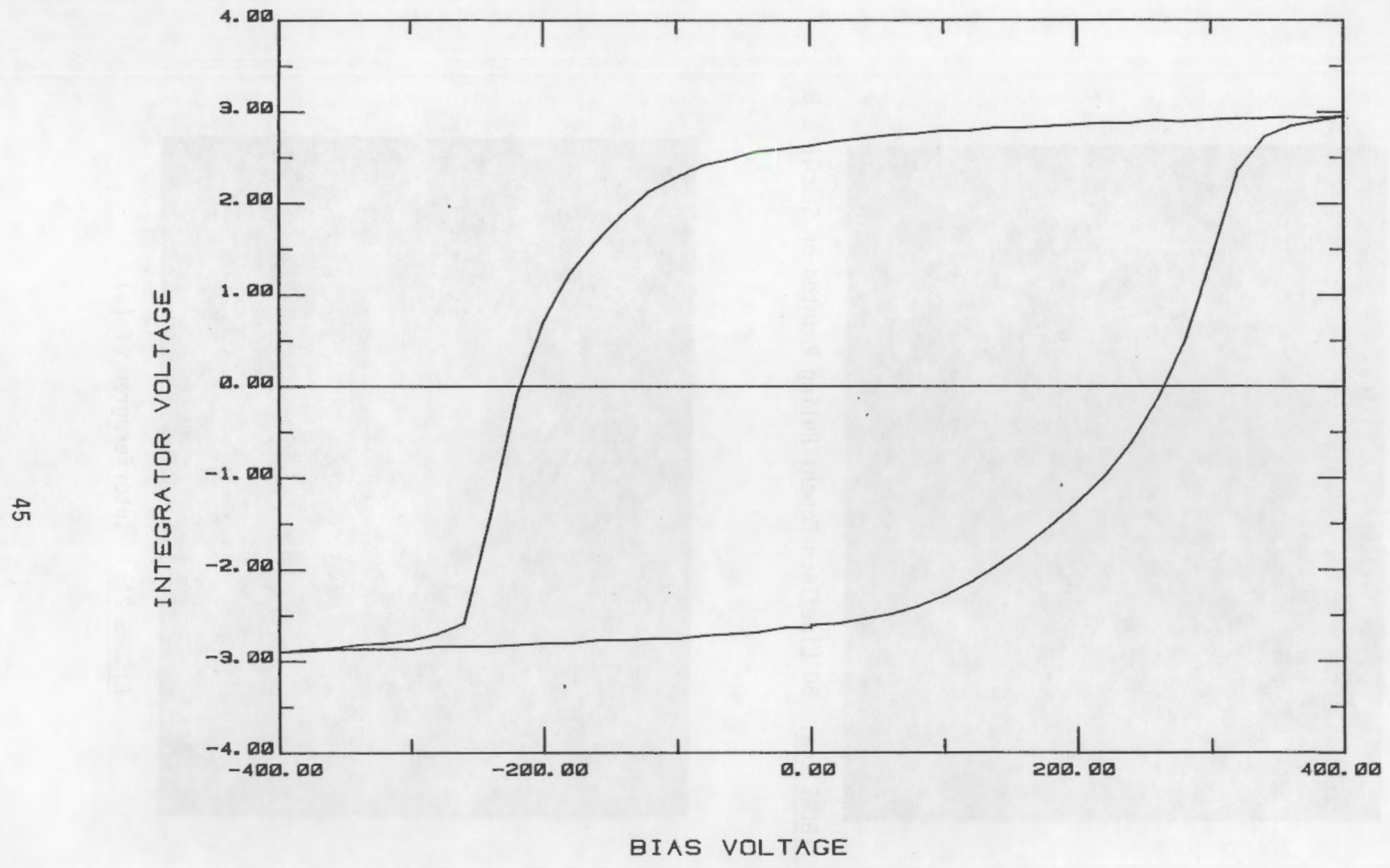

FIGURE 23. $0_{2}^{+}$Implanted Sample B-8, First Characteristic Curve, File 022 


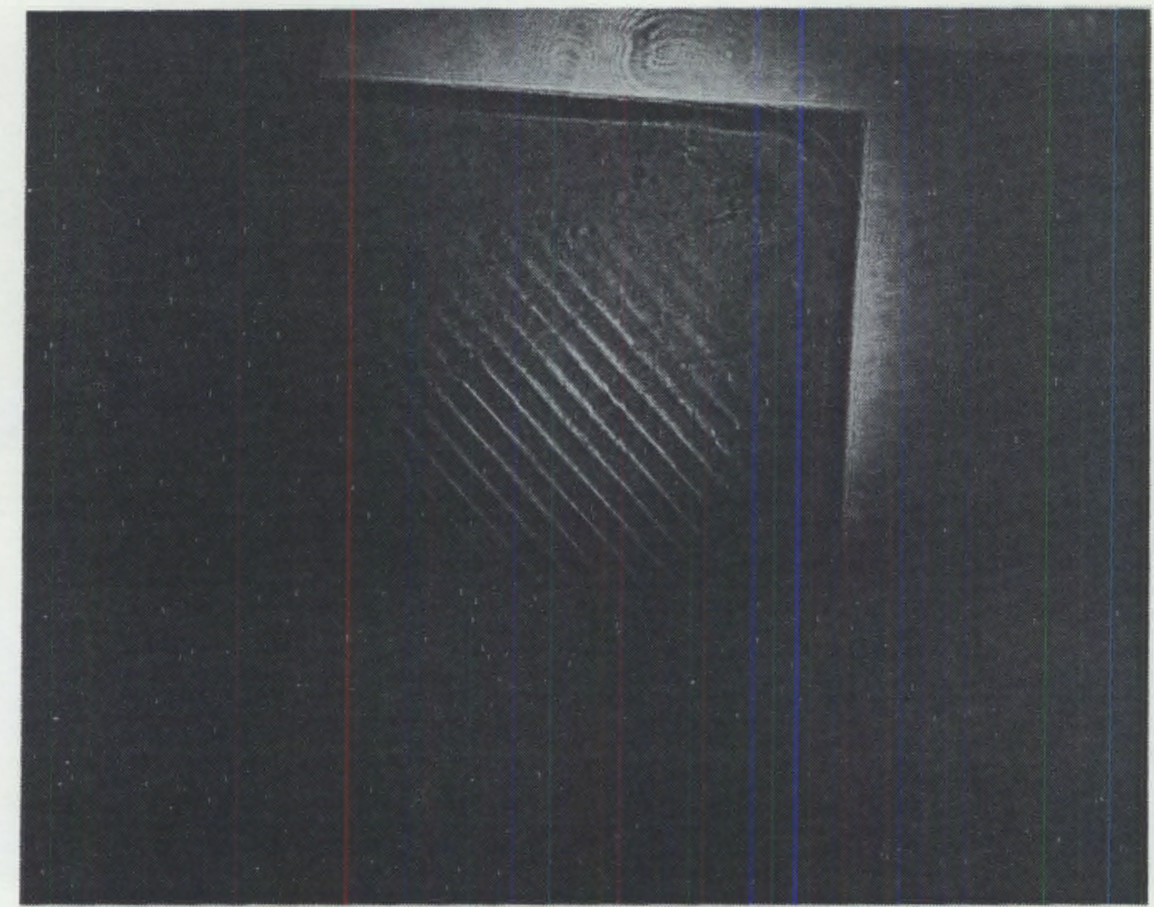

FIGURE 24a. 50 Line/Inch Ronchi Ruling Printed on Sample B-8

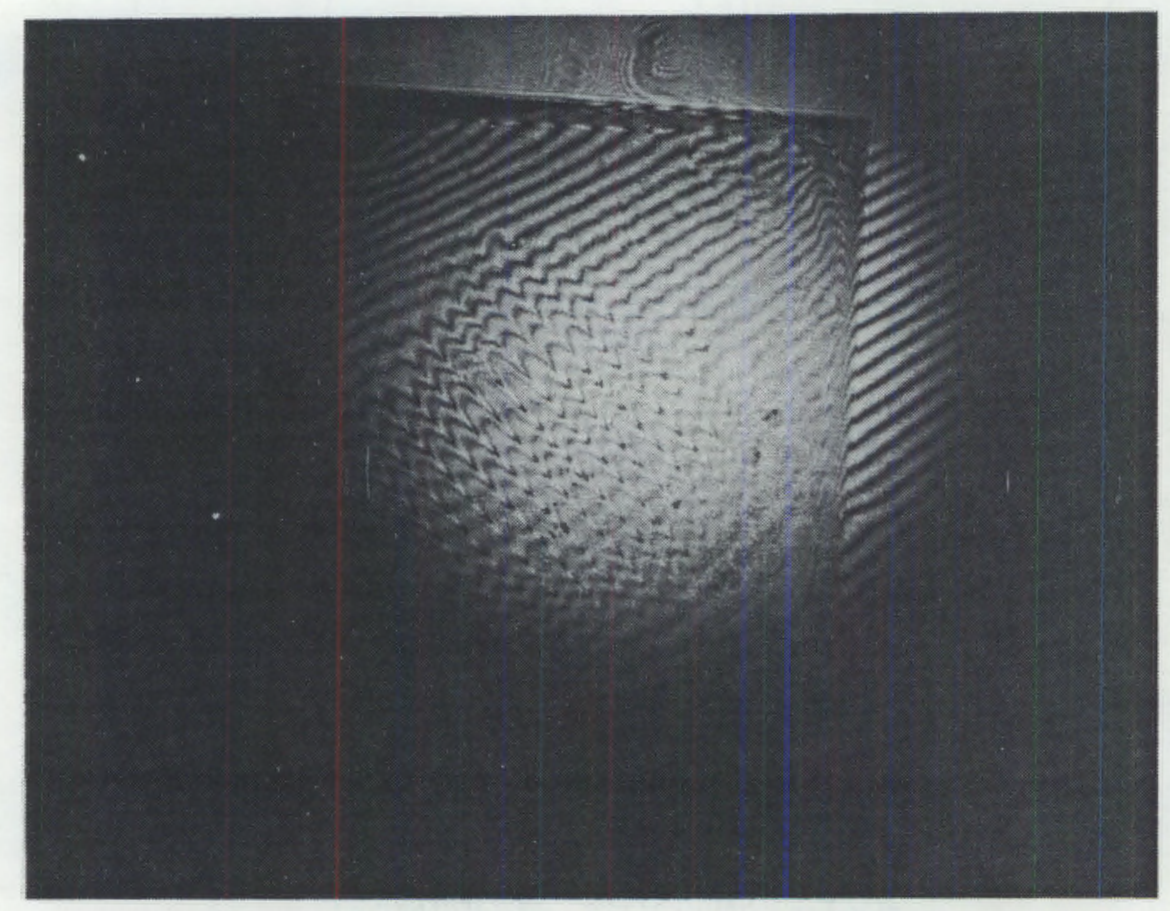

FIGURE 24b. Interferogram of (a) 


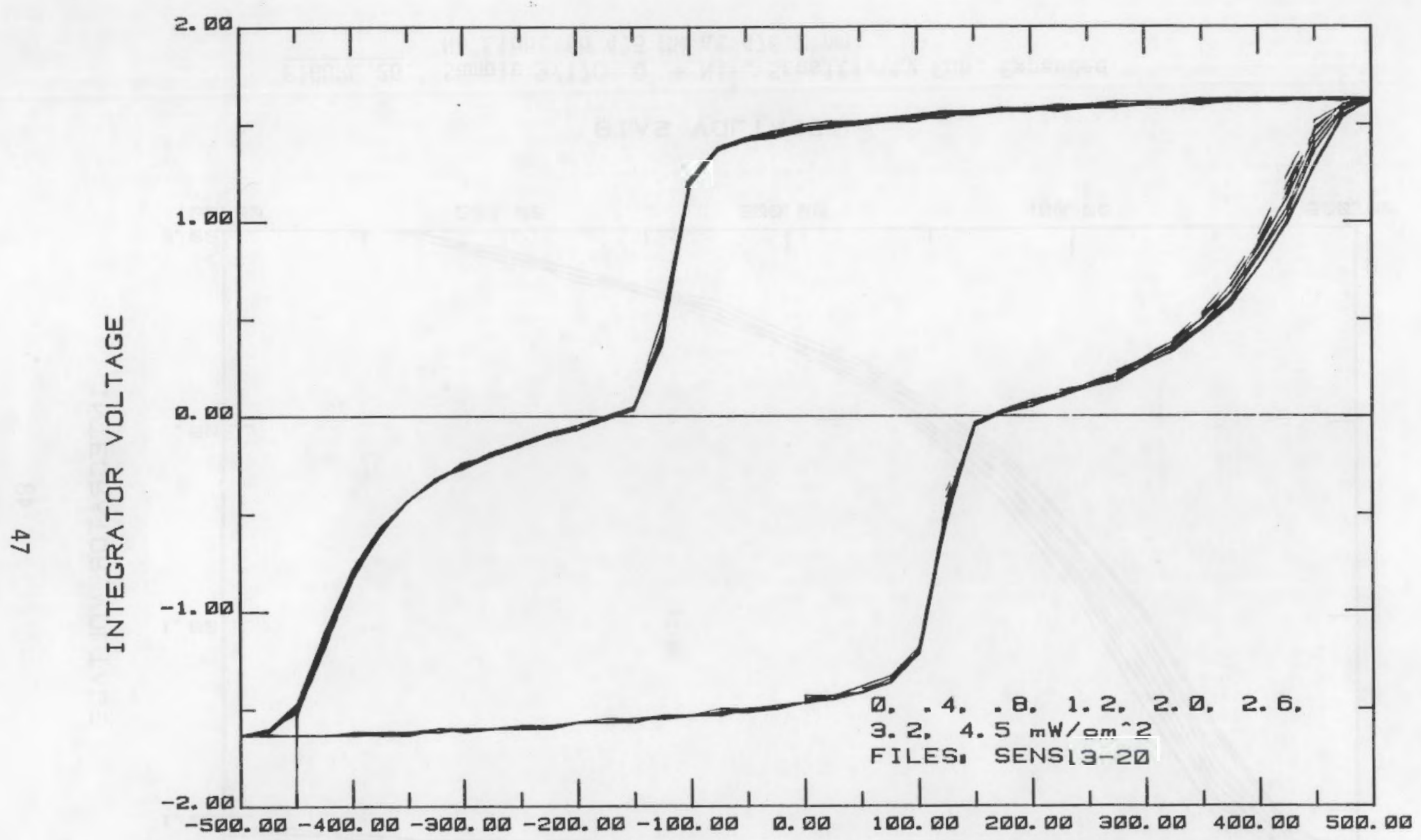

BIAS VOLTAGE

FIGURE 25. Sample $9 / 17 \mathrm{C}, \mathrm{O}^{+}+\mathrm{Ni}^{+}$, Sensitivity Run No Light to $4.5 \mathrm{MW}$ at $476.2 \mathrm{~nm}$ 


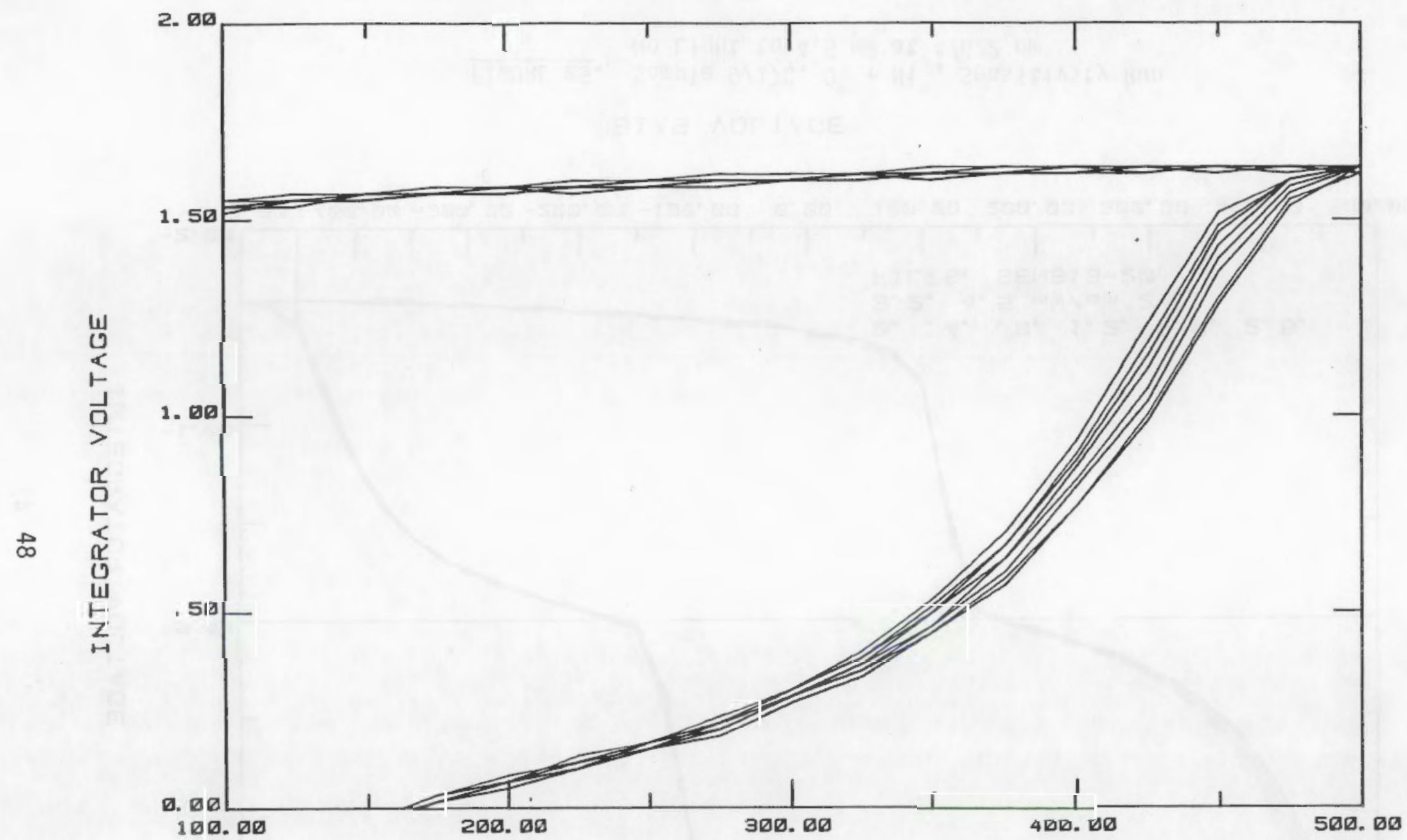

BIAS VOLTAGE

FIGURE 26. Sample $9 / 17 \mathrm{C} \mathrm{O}^{+}+\mathrm{Ni}^{+}$, Sensitivity Run, Expanded No Light to $4.5 \mathrm{MW}$ at $476.2 \mathrm{~nm}$ 


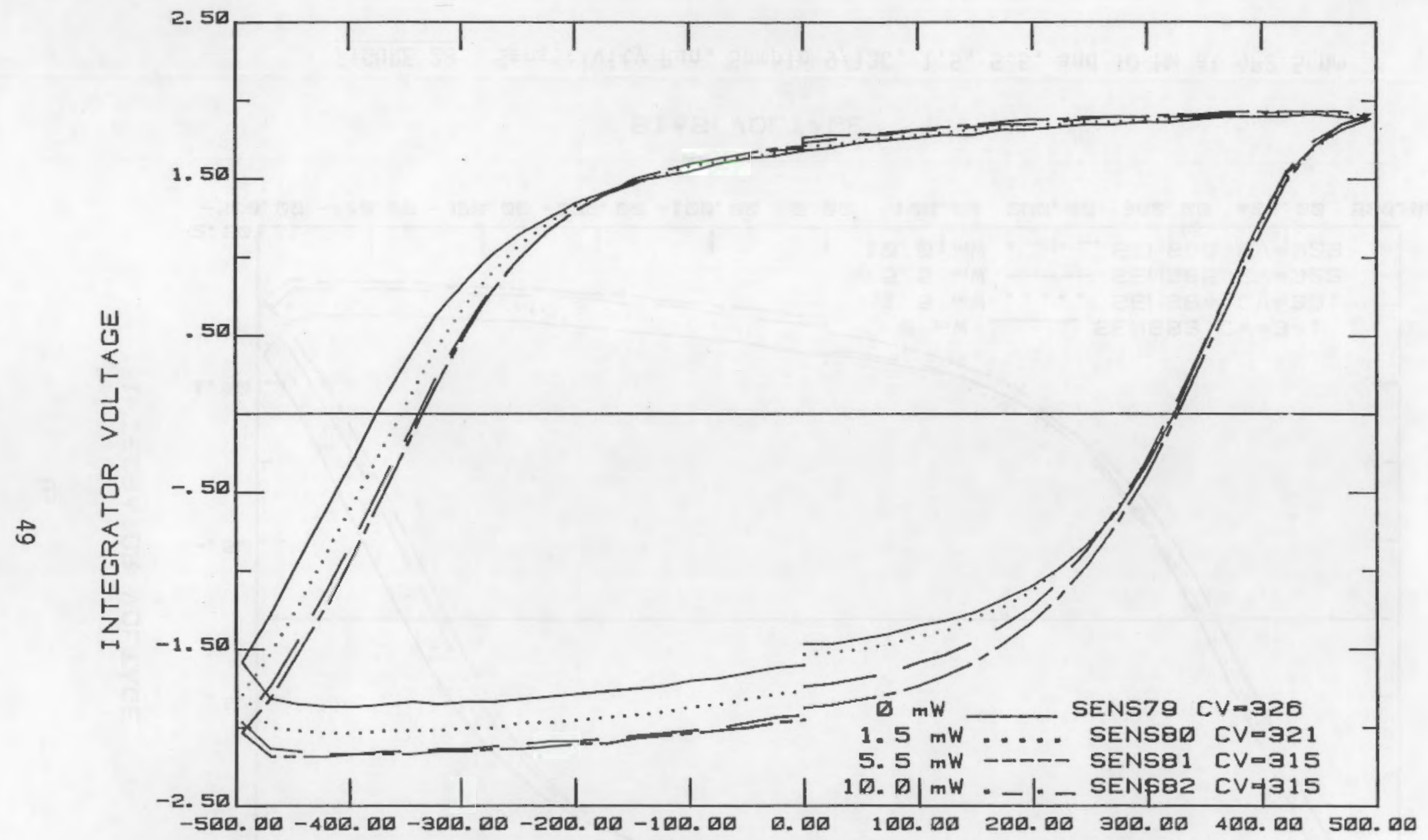

BIAS VOLTAGE

FIGURE 27. Sensitivity Run, Sample $9 / 13 \mathrm{C}, 1.5,5.5$, and $10 \mathrm{MW}$ at $476.2 \mathrm{~nm}$ 


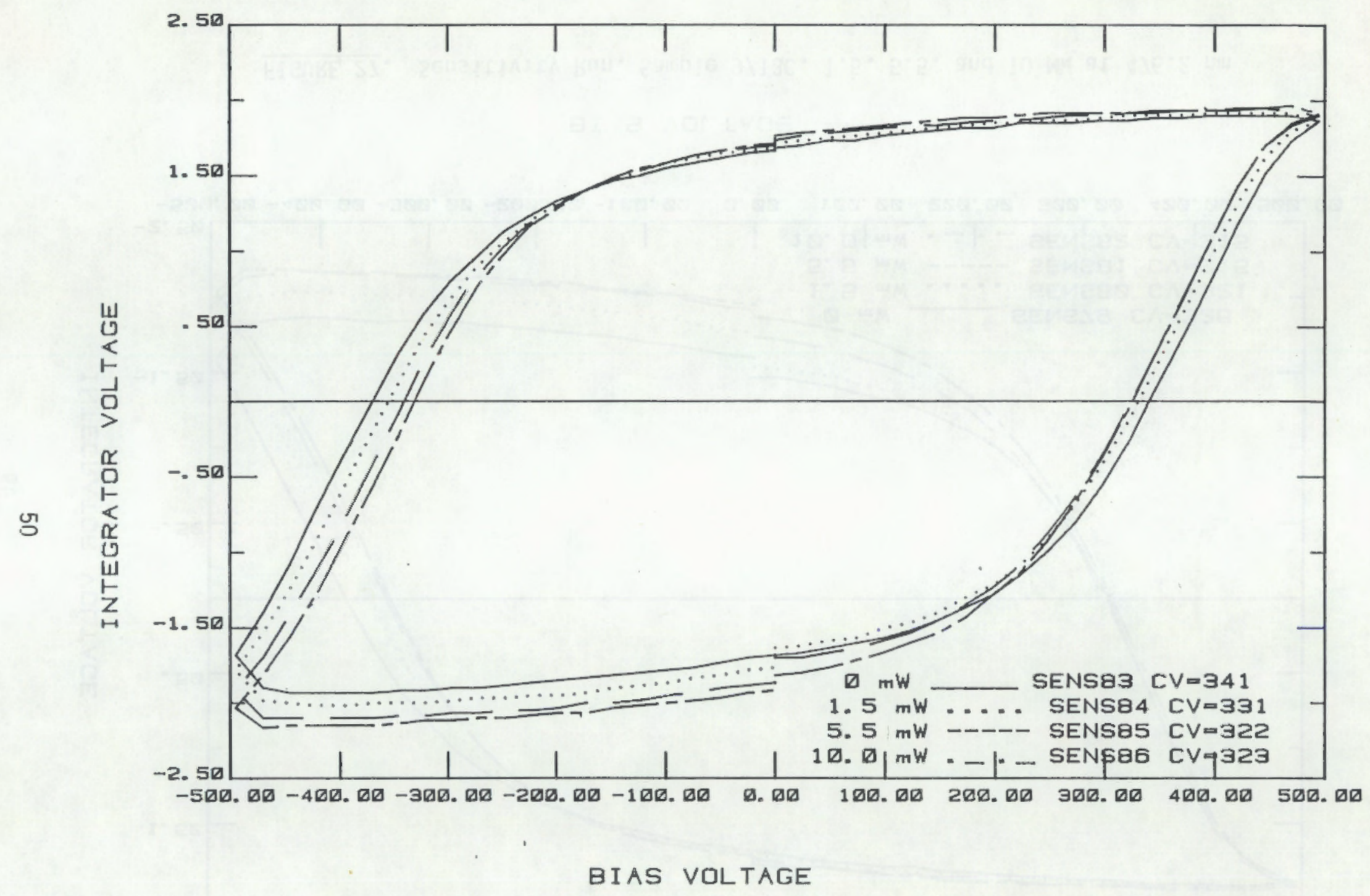

FIGURE 28. Sensitivity Run, Sample $9 / 13 \mathrm{C}, 1.5,5.5$, and $10 \mathrm{MW}$ at $482.5 \mathrm{~nm}$ 



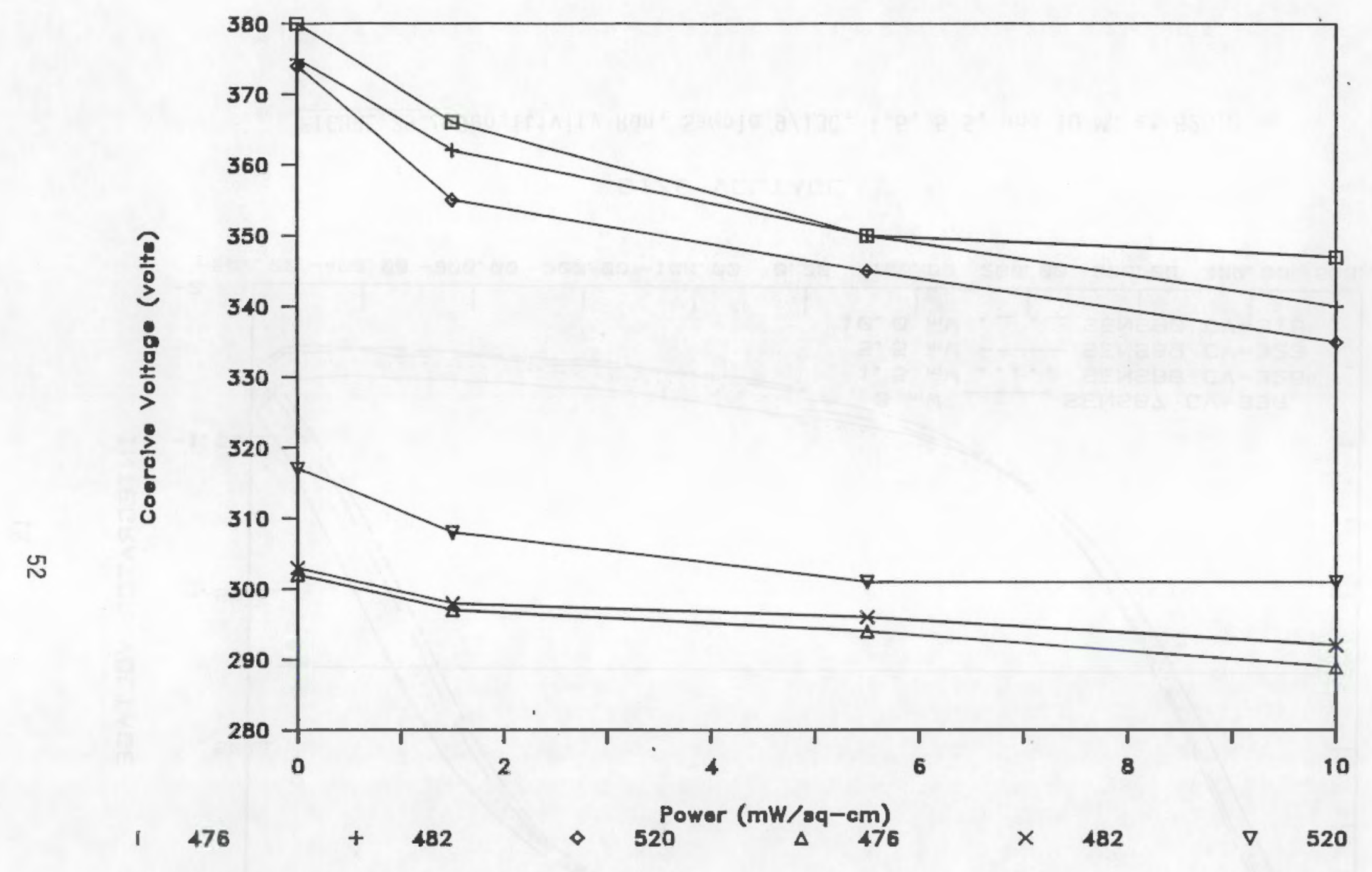

FIGURE 30. USAF PLZT Adaptive Optics - Sensitivity: $9 / 13 \mathrm{C}(\mathrm{a})$ and 9/22B 


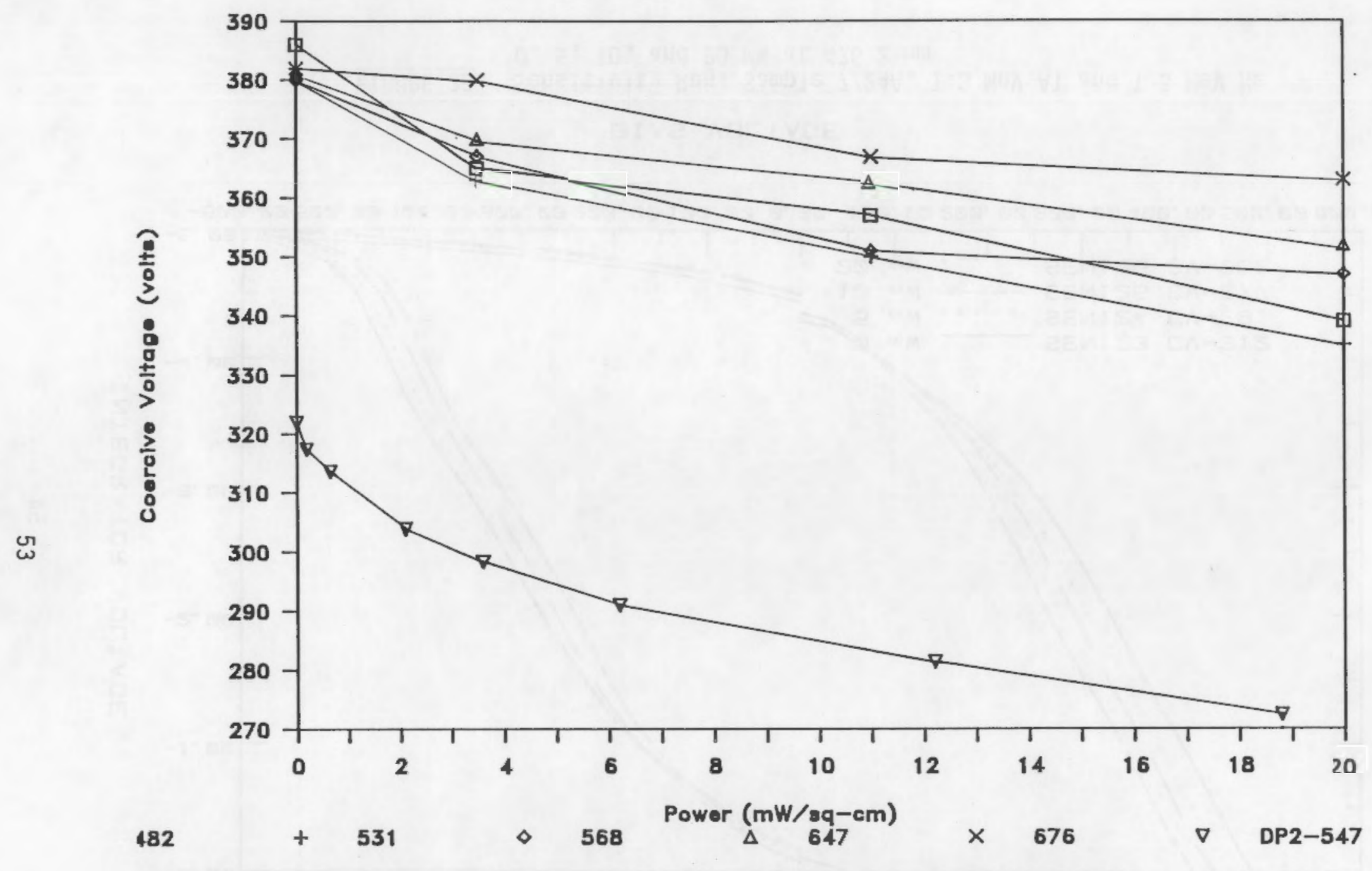

FIGURE 31. USAF PLZT Adaptive Optics - Sensitivity: $9 / 13 C(e)$ and DP2-547 


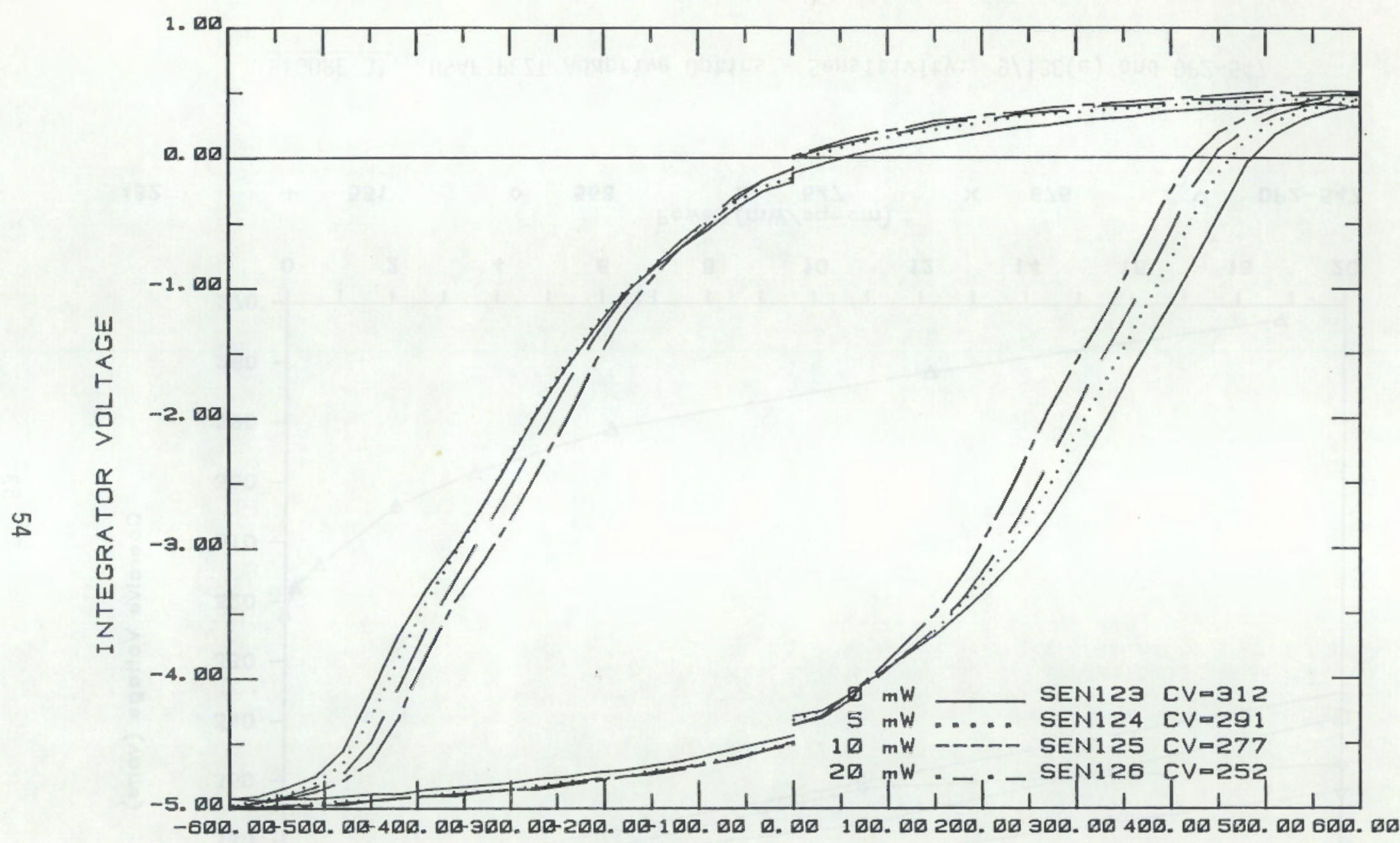

BIAS VOLTAGE

FIGURE 32. Sensitivity Run, Sample $7 / 24 \mathrm{~A}, 1.3 \mathrm{MeV} \mathrm{A1}$ and $1.3 \mathrm{MeV} \mathrm{Ne}$ $0,5,10$, and $20 \mathrm{MW}$ at $476.2 \mathrm{~nm}$ 
for reference, and the summary plot for the sample showing change in coercive voltage as a function of wavelength is shown in Figure 33.

The previous sample was then annealed for one hour at $300^{\circ} \mathrm{C}$ in air, and - a set of sensitivity data taken. Figure 34 shows the data for $476 \mathrm{~nm}$ for reference, and Figure 35 shows the summary curve. Notice the difference in the shape of the curve and that only zero and $20 \mathrm{~mW}$ exposures were used. Also notice in the summary curve that the sensitivity has dropped overall, but that there is also a single anomalous point.

In August, the second set of $7 / 70 / 30$ samples was completed with a neon/chromium implant. Sample $7 / 230$ was characterized. Again, Figure 36 for a wavelength of $476 \mathrm{~nm}$ is shown for reference, and Figure 37 shows the summary. It is important to note that in this case, the vertical axis is the change in coercive voltage per unit change in recording beam intensity. The result is that these numbers should be multiplied by $20 \mathrm{~mW}$ to be directly comparable to those in previous figures. In this case, the data was extended to $1500 \mathrm{~nm}$ by using an arc lamp for wavelengths beyond $676 \mathrm{~nm}$. From this plot, it is clear that the sensitivity is gone at approximately $1000 \mathrm{~nm}$.

Characterization of the triple implanted nickel samples, series $9 / 20$, was attempted; but it was clear that the saturation voltage would not be reachable without taking special care to avoid arcing. Figure 38 shows the characteristic curves at zero and $50 \mathrm{~mW}$ at $476 \mathrm{~nm}$. The curve represents a maximum applied voltage of 500 volts. Figure 39 shows the equivalent curve at $676 \mathrm{~nm}$. Further attempts to characterize these implants will be made in FY87.

\section{PROPOSED FY87 TASKS}

The research tasks proposed for FY87 include continuation of FY86 tasks, plus a theory/modeling task and some revised emphases resulting from the progress made during FY86 and the changing needs of the sponsor. These tasks are shown in Table 7 entitled "FY86 PROPOSED TASKS," and formed the basis for the draft proposal submitted to the sponsor under a cover letter dated 7 August 1986. The tasks and breakdown in Table 7 include all tasks in the draft proposal, but the actual scope is to be negotiated. 




FIGURE 33. USAF PLZT Adaptive Optics - Sensitivity: 7/24A 



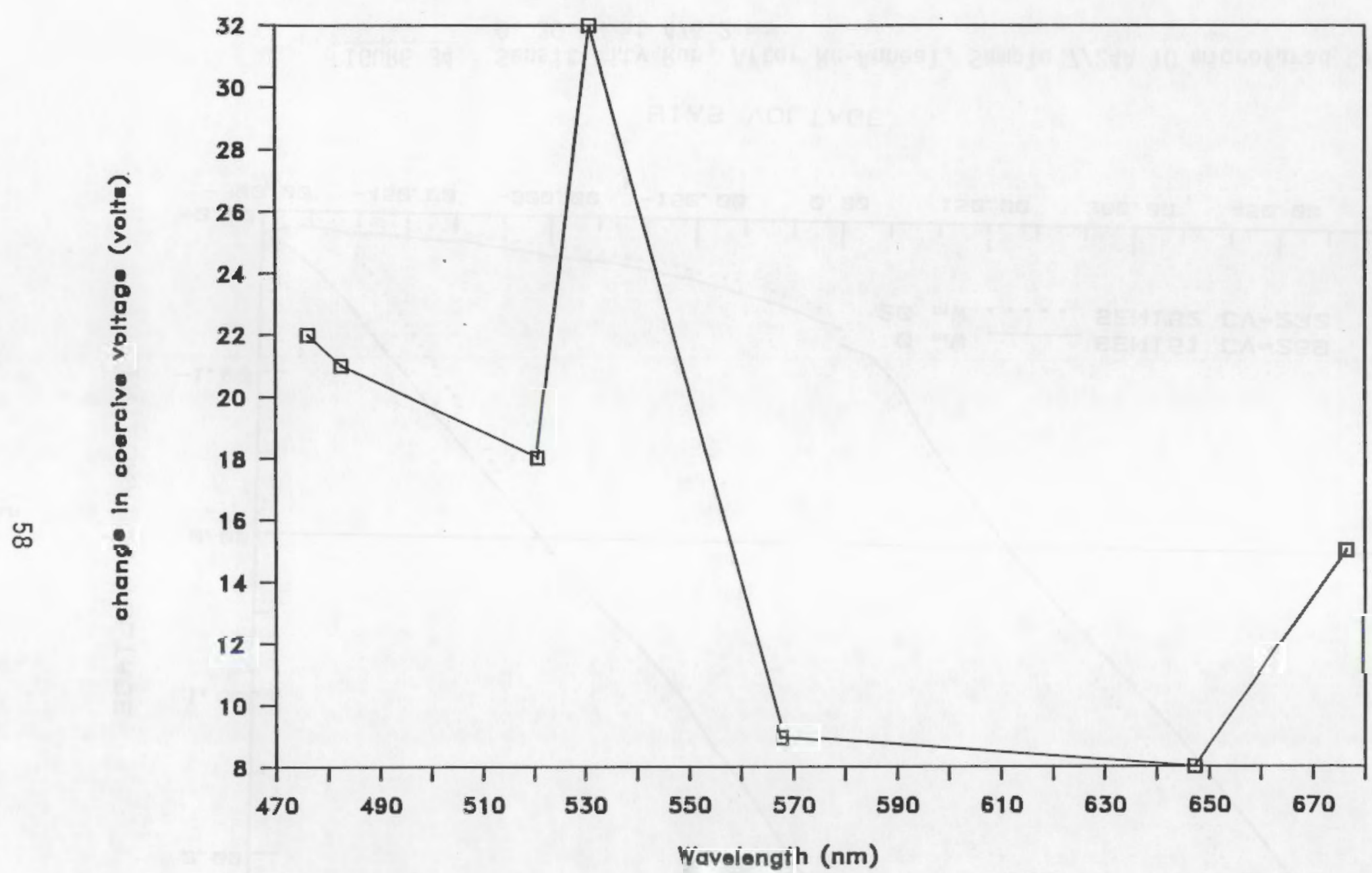

FIGURE 35. USAF PLZT Adaptive Optics - Sensitivity After Anneal: 7/24A 


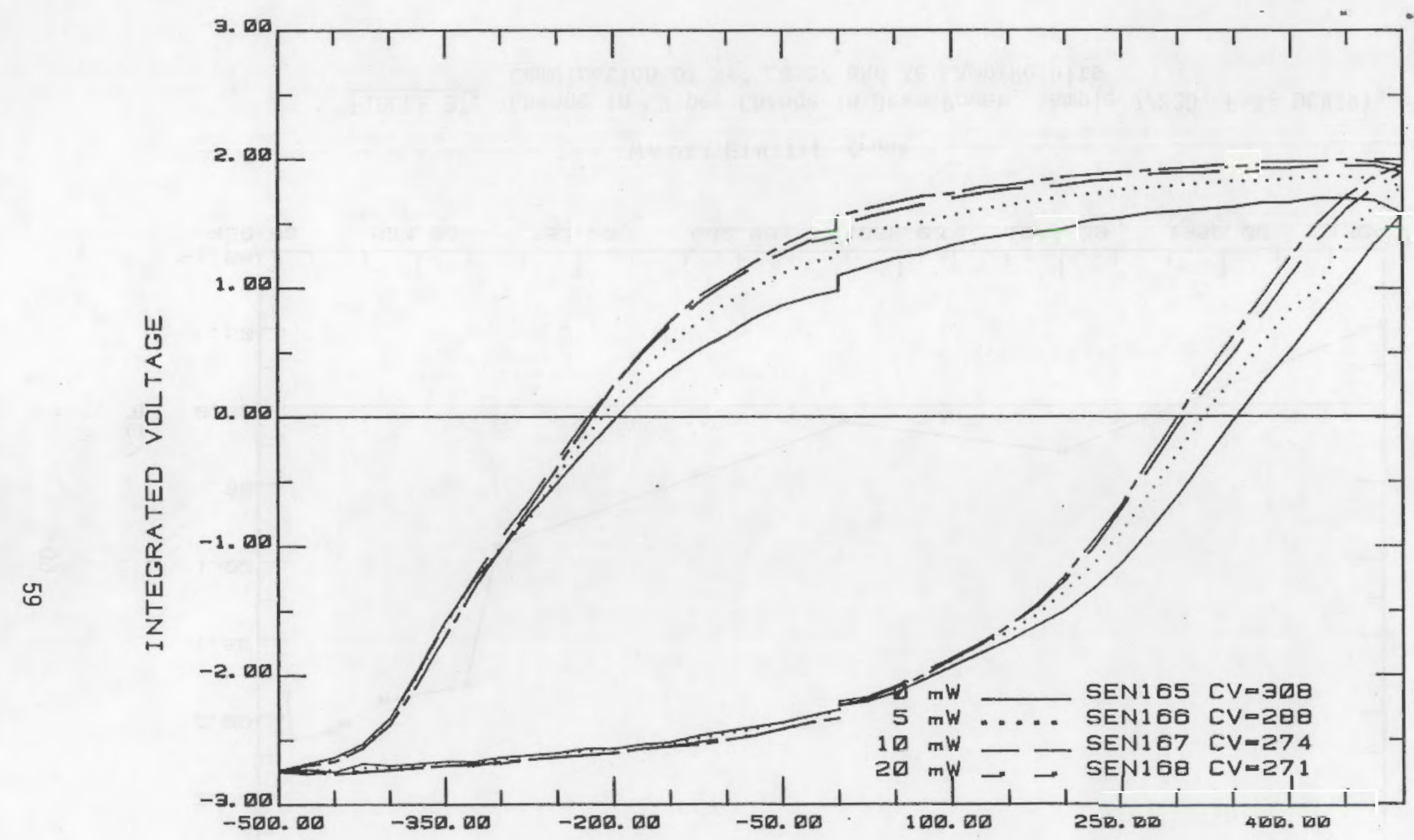

BIAS VOLTAGE

FIGURE 36. Sensitivity Run, \#7/23D, 7/70/30 $1.3 \mathrm{MeV} \mathrm{Ne}$ and $1.3 \mathrm{MeV} \mathrm{Cr}$, $0,5,10$, and $20 \mathrm{MW}$ at $476.2 \mathrm{~nm}$ 


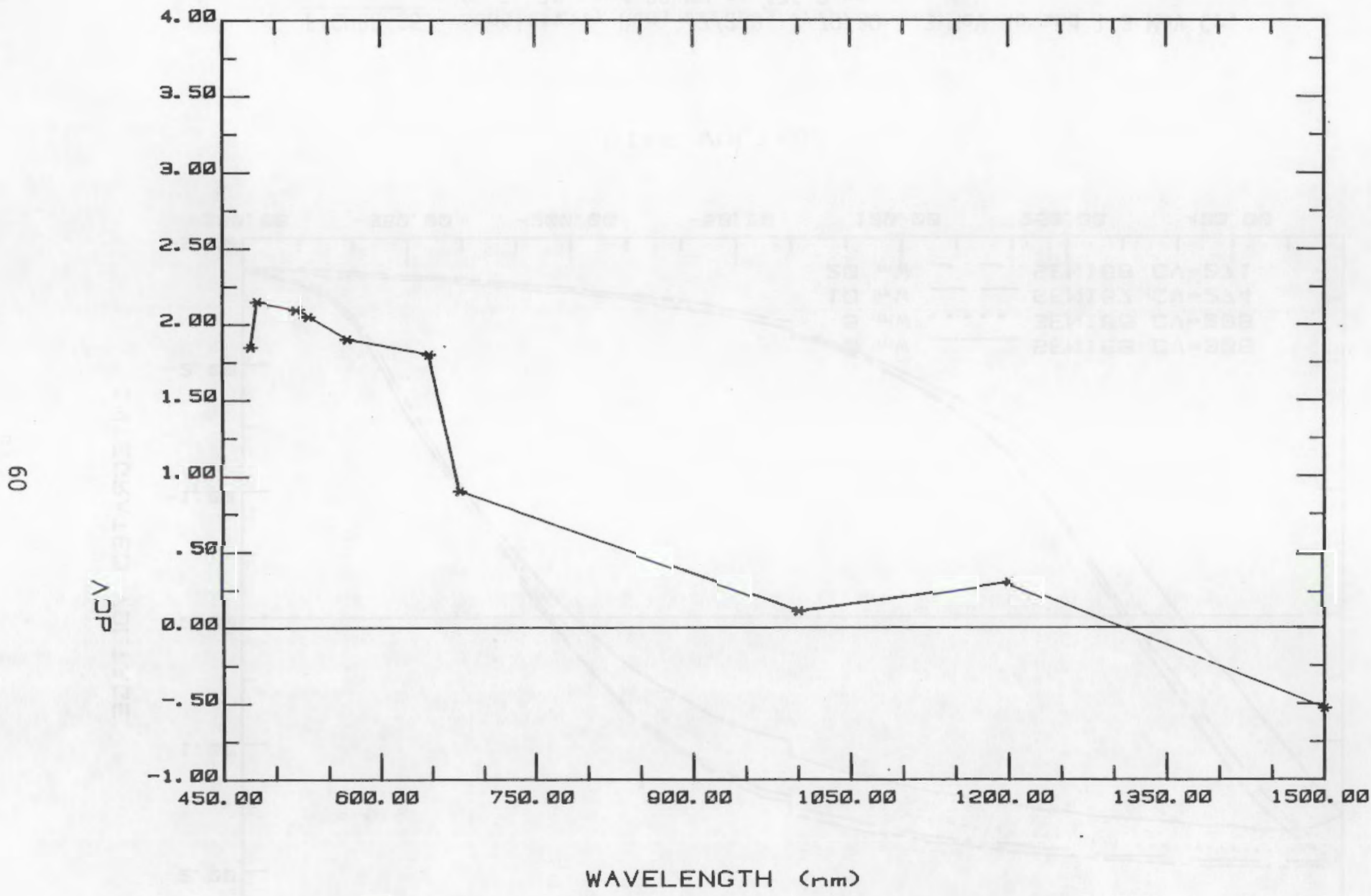

FIGURE 37. Change in CV per Change in Beam Power, Sample 7/23D, File DCVIR1, Combination of $\mathrm{Kr}^{+}$Laser and Xe Lamp Results 


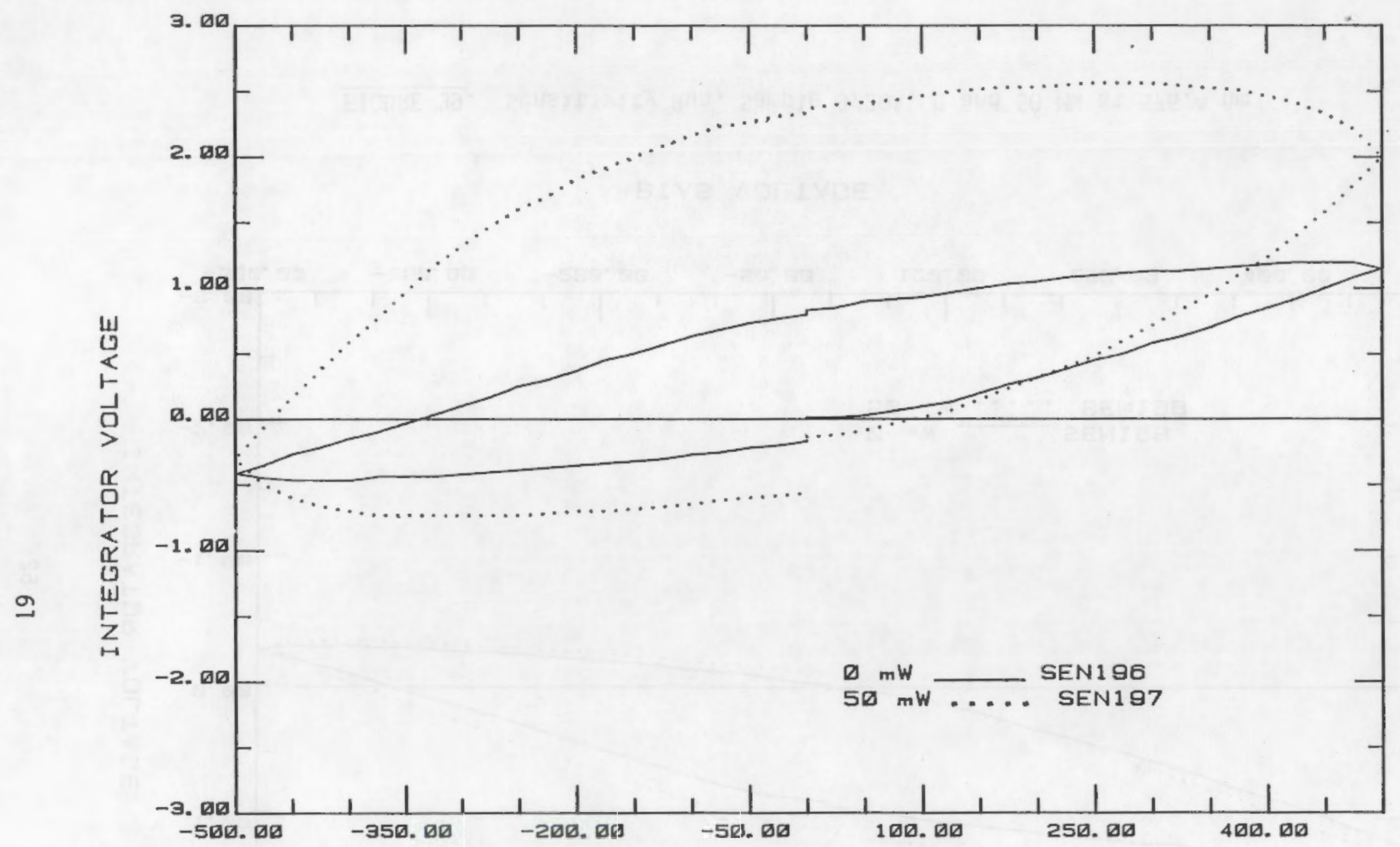

BIAS VOLTAGE

FIGURE 38. Sensitivity Run, Sample $9 / 20 \mathrm{~A}, 0$ and $50 \mathrm{MW}$ at $476.2 \mathrm{~nm}$ 


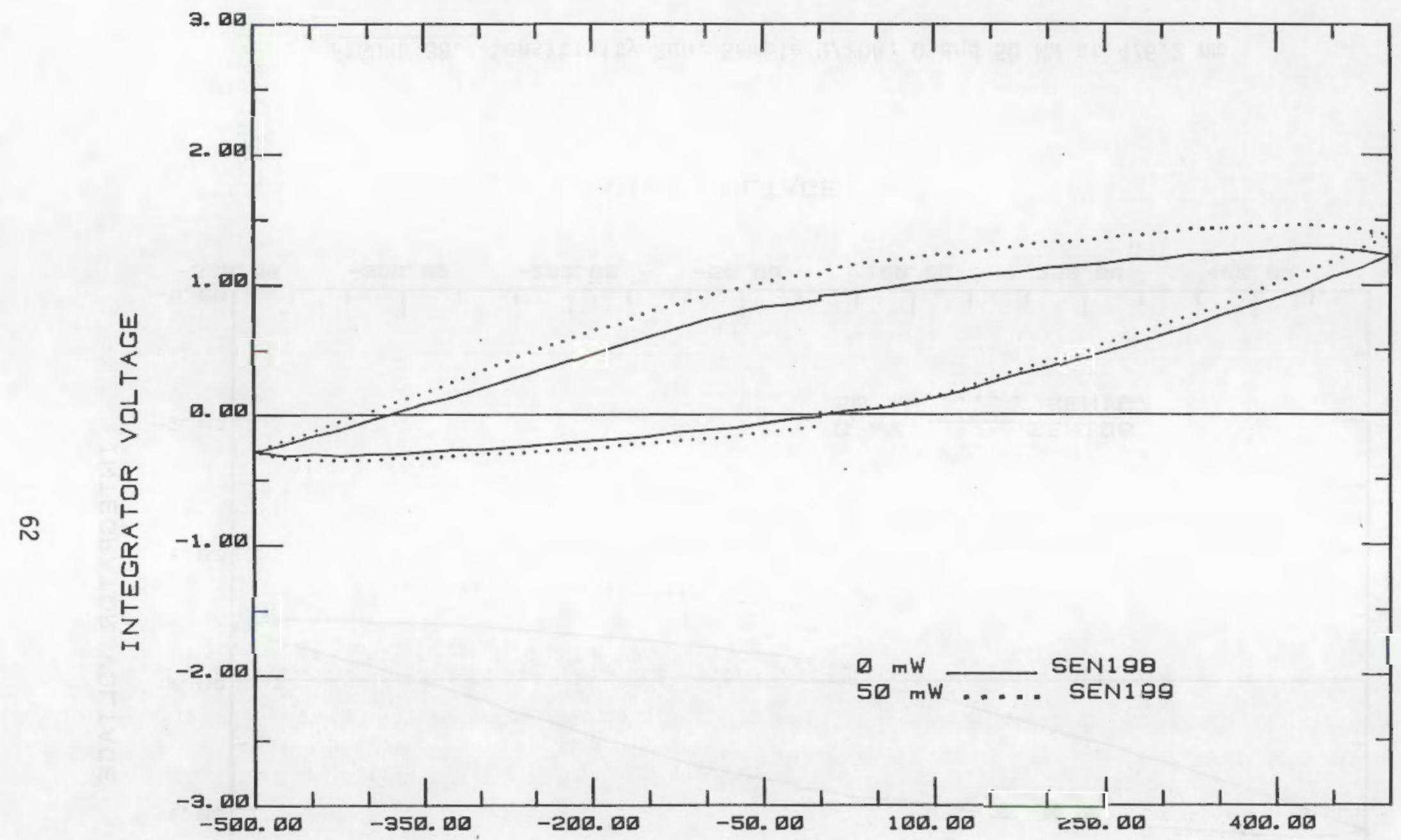

BIAS VOLTAGE

FIGURE 39. Sensitivity Run, Sample $9 / 20 \mathrm{~A}, 0$ and $50 \mathrm{MW}$ at $676.4 \mathrm{~nm}$ 
TABLE 7.

FY 87 PROPOSED TASKS

\subsection{Ion Implantation}

1.1 routine implantation to provide the sponsor with samples for his research

1.2 new implantations according to Land's recomnendations to confirm ions and parameters

1.3 implant multiple energy/fluence nickel and/or other active fons to give a uniform damage profile to over 1 micrometer

1.4 implant nitrogen and/or oxygen at multiple energies and fluences to provide a uniform damage profile to over 1 micrometer, and result in the ions being deposited at discrete depths

1.5 implant active ions or active plus inert ions to greater than 3 micrometers

1.6 implant inert ions following thermal diffusion of various active ions

\subsection{Coatings}

2.1 provide routine coating services for all implantation

2.2 develop the process for deposition and structural characterization of thin film PLZT

2.3 continue investigating stress curvature effects as needed

\subsection{Characterization}

3.1 continue routine characterization

3.2 expand characterization to include measurement of MTF

3.3 investigate methods of reporting photosensitivity which are recognized in the technical community yet meaningful for this work

3.4 investigate reflective phase conjugation

\subsection{Modeling}

4.1 initiate studies to refine the Peercy-Land model to reflect results of PNL implantations

4.2 Initiate studies to form the basis for processing of thin film PLZT for enhanced photosensitivity 


\section{REFERENCES}

Glang, R., R. A. Holmwood, and R. L. Rosenfield. "Determination of Stress in Thin Films on Single Crystal Substrates." Review of Scientific Instruments, $36(1): 7$.

Hoffman, R. W. "Mechanical Properties of Non-Metallic Thin Films," in Physics of Non-Metallic Thin Films, p. 309.

Jacobsson, R. 1975. "Inhomogeneous and Coevaporated Homogeneous Films for Optical Applications," in Physics of Thin Films, Volume 8.

Manifacier, J. C., J. Gasiot, and J. P. Fillard. 1976. "A Simple Method for the Determination of the Optical Constants $n, k$ and the Thickness of a Weakly Absorbing Thin Film." Journal of Physics, E9, 1002.

Pawlewicz, W. T., P. M. Martin, R. W. Knoll, and I. B. Mann. 1986. "Multilayer Optical Coating Fabrication by DC Magnetron Reactive Sputtering Techniques." To appear to SPIE Proceedings, Volume 678.

van der Pauw, L. J. 1958. A Method of Measuring Specific Resistivity and Hall Effect of Discs of Arbitrary Shape. Philips Research Reports 13, 1. 
No. of

Copies

Offsite

2 Cpt. J. A. Bullington

Air Force Weapons Laboratory

Conventional Adaptive Optics Branch

Kirtland Air Force Base NM 87117-6008

Ms. S. D. Mancha

Air Force Weapons Laboratory

Conventional Adaptive Optics Branch

Kirtland Air Froce Base NM 87117-6008

2 DOE Technical Information Center

Onsite

DOE Richland Operations office

J. J. Sutey

14 Pacific Northwest Laboratory

C. R. Batishko

J. L. Brimhal1

R. A. Craig

W. T. Pawlewicz

K. A. Stahl

L. H. Toburen

Project File

Publishing Coordination (5)

Technical Report Files (2) 
